\title{
Colonoscopic screening is associated with reduced Colorectal Cancer incidence and mortality: a systematic review and meta-analysis
}

\author{
Jiaxin Zhang ${ }^{1,2^{*}}$, Guang Chen ${ }^{1,2^{*}}$, Zhiguo Li1,2, Peng Zhang ${ }^{1,2}$, Xiaoke Li ${ }^{1,2}$, Da'nan Gan ${ }^{1,2}, X^{\prime}$ Cao ${ }^{1,2}$, Hongbo \\ Du $^{1,2}$, Jiaying Zhang 3 , Ludan Zhang ${ }^{1,2}$, Yong' an Ye $\mathrm{Y}^{1,2}$ \\ 1. Dongzhimen Hospital, Beijing University of Chinese Medicine, Beijing, China. \\ 2. Institute of Liver Diseases, Beijing University of Chinese Medicine. \\ 3. Ministry of Education Key Laboratory of Bioinformatics, Tsinghua-Peking Center for Life Sciences, School of Life Sciences, Tsinghua University, Beijing \\ 100084, China. \\ *These authors contributed equally to this work. \\ $\bowtie$ Corresponding author: Yong'an Ye, E-mail: yeyongan@vip.163.com.
}

(C) The author(s). This is an open access article distributed under the terms of the Creative Commons Attribution License (https://creativecommons.org/licenses/by/4.0/). See http:/ /ivyspring.com/terms for full terms and conditions.

Received: 2020.04.04; Accepted: 2020.08.03; Published: 2020.08.15

\begin{abstract}
It is the great priority to detect colorectal cancer (CRC) as early as possible, finally to reduce the incidence and mortality of CRC. However, although colonoscopy is recommended in many consensuses, yet no one systematic review is conducted to figure out how colonoscopy could change the incidence and mortality. In our study, we conducted a comprehensive meta-analysis to evaluate the association between colonoscopy screening and the incidence or mortality of CRC. PubMed, EMBASE, and PMC database were systematically searched from their inception to June 2020. A total of 13 cohort and 16 case-control studies comprising $4,713,778$ individuals were obtained in this review. Our results showed that colonoscopy was associated with a $52 \% R R$ reduction in incidence of CRC ( $R R: 0.48,95 \% \mathrm{Cl}: 0.46-0.49)$ and $62 \% R R$ reduction in mortality of CRC (RR: $0.38,95 \% \mathrm{Cl}$ : 0.36-0.40). Subgroup analysis of different interventions, study design, country, sample size, age or sex showed that the incidence and mortality reduction remained consistent, and colonoscopy screening had the same effect on people below and above 50. Our study indicated that colonoscopy could significantly reduce the incidence and mortality of CRC.
\end{abstract}

Key words: colorectal cancer; colonoscopy; incidence and mortality; meta-analysis

\section{Introduction}

Colorectal cancer (CRC), one of the most common malignancies [1], is the leading cause of cancer death worldwide $[2,3]$ and the second most common cause of cancer death in the United States [4]. However, more than $85 \%$ of the CRC are found to be advanced; thus, their 5-year survival rate is poorly $50 \%$ [3], even though both surgery, chemotherapy, and targeted therapy are used actively. Hence, it is the great priority to detect CRC as early as possible, finally to reduce the incidence and mortality of CRC. However, the diagnosis rate of early CRC in China is less than $10 \%$ [5].

In order to detect CRC earlier, the American Cancer Society recommends screening for colorectal cancer from the age of 45 , based on epidemiological data and mathematical models. Both the fecal occult blood test (FOBT), fecal DNA test and colonoscopy are the mainstream detecting methods. Compared with the other two methods, colonoscopy is the gold standard for the diagnosis of CRC, and it could meanwhile provide an opportunity for treatment [6, 7]. Although several studies have shown that endoscopy could reduce the incidence and mortality of CRC [8-14], and colonoscopy is strongly recommended to prevent CRC by early detection of cancer in the Asia Pacific Consensus [15], yet the quality of evidence is II-2, and the classification of recommendation is $B$ due to the fact that sample size 
of the original studies supporting the evidence is relatively small, and the strong large-scale randomized trials are still ongoing [16-18].

Although it is recommended in the consensus, colonoscopy screening programs have not been widely implemented in many European countries [19, 20] and Asia-Pacific regions [15]. Even in the United States and Germany where screening programs started in the very early years, the screening rate was only $54 \%$ by 2013 [21] and $20 \sim 30 \%$ by 2012 [22] respectively. Apart from the high costs and lack of colonoscopy professionals, another reason is that actually no one systematic review is conducted to figure out how colonoscopy could change the incidence and mortality. As a result, neither the doctors nor the patients could consider about the balance between the potential benefits and harms of receiving colonoscopy. Recently many case-control and cohort studies based on larger-scale data have reported that colonoscopy might reduce CRC mortality [23-26] in patients with left-sided colon cancer $[8,27]$. Therefore, this systematic review and meta-analysis was conducted to evaluate the association between colonoscopy screening and the incidence or mortality of CRC.

\section{Materials and Methods}

This systematic review and meta-analysis was conducted on the basis of the Meta-Analysis of Observational Studies in Epidemiology (i.e., MOOSE) and Preferred Reporting Items for Systematic Reviews and Meta-analysis (PRISMA) statement [28, 29]. The protocol has been registered at PROSPERO (CRD42019122795, http://www.crd.york.ac.uk/ PROSPERO).

\section{Search Strategy}

A comprehensive electronic literature search was performed on PubMed, EMBASE and PMC databases from inception through June 2020 with the following terms: "colonoscopy or colonoscopy" AND "Colorectal Neoplasm or Colorectal Tumor or Colorectal Carcinoma or Colorectal Cancer or Intestinal Neoplasm or Intestinal Cancer or Gastrointestinal Neoplasm or Gastrointestinal Cancer" AND "relative risk or relative risks or odds ratio or odds ratios or rate ratio or rate ratios or risk ratio or risk ratios or hazard ratio or hazard ratios or ratio" AND "case-control studies or cohort studies or cohort or case-control". The detailed search strategy was described in Supplementary Tables 1-3. What's more, we reviewed the references of identified studies for further study. The authors examined the titles and abstracts independently and in duplicate to identify studies that might be eligible and then reviewed the full text to determine trials that met the eligibility criteria.

\section{Inclusion Criteria and Exclusion criteria}

Observational studies (prospective cohort, retrospective cohort, nested case-control, or case-control studies) were included if they met the following criteria: (1) A general population older than 18 years old who are not diagnosed with CRC. (2) The control group did not receive colonoscopy or other examination methods. (3) CRC incidence or mortality confirmed by pathologic diagnosis, and reported risk estimates, such as hazard ratios (HRs), relative risks (RRs), or odds ratios (ORs) with corresponding 95\% confidence intervals $(\mathrm{CIs})$ or sufficient data for their estimation. (4) Cohort (prospective or retrospective), or case-control studies. As for exclusion criteria, they were displayed as follows: (1) colonoscopy was assessed only in patients with premalignant conditions, colorectal adenoma, inflammatory bowel disease (IBD), ulcerative colitis (UC) and Crohn disease (CD); (2) Non-English published studies; and (3) protocol, case reports, comments, reviews, expert opinions, conference abstracts, letters, and animal experiments.

\section{Data Extraction and Quality Assessment}

For all included studies, the following information was extracted: first author, publication year, design, period, country, sample size, gender, age, intervention, follow-up duration, comparator, frequency and timing of colonoscopic screening, adjustments or matching factors, numbers of outcomes, and effect estimates. The primary outcome was the incidence or mortality of CRC. The quality of each study was evaluated with the Newcastle-Ottawa Quality Rating Scale (NOS), as one of the most useful scales to evaluate the quality of non-randomized studies (http://www.ohri.ca/programs/clinical_ epidemiology/oxford.htm). If any disagreement achieved, a third reviewer would join in and reached a consensus.

\section{Statistical Analysis}

Considering low heterogeneities within and between studies, we used the fixed-effects model [30] to calculate the study-specific $R R$ estimates. $R R$ was employed as a common measure of the association between colonoscopic screening use and the incidence and mortality of CRC. To simplify the terminology, the effect estimates of ORs from case-control studies were directly regarded as an estimate of relative risk $(R R)$. Cochrane's Q statistic $(p<0.10$ suggesting significant heterogeneity) and the $I^{2}$ statistic $\left(I^{2}>75.0 \%\right.$ representing substantial heterogeneity, $50.0 \% \leq I^{2} \leq 75.0 \%$ representing moderate 
heterogeneity and $I^{2}<50 \%$ representing low heterogeneity) were adopted to qualitatively and quantitatively evaluate heterogeneity across studies, respectively [31]. Sensitivity analysis was conducted by omitting each study in turn. Using Begg's and Egger's test to quantitatively detect publication bias, and the significance level was $p \leq 0.1[32,33]$. If publication bias was significant, the robustness of meta-analysis results was verified by the trim and fill method [34]. All statistical analyses were performed using Stata version 12.0 (StataCorp, College Station, Texas, USA). The statistical significance level was set at a two-sided $p<0.05$ unless otherwise specified.

\section{Results}

A total of 3,536 studies were included, as is shown in Figure 1. After the deletion of duplicate studies, there are 2,614 records were considered

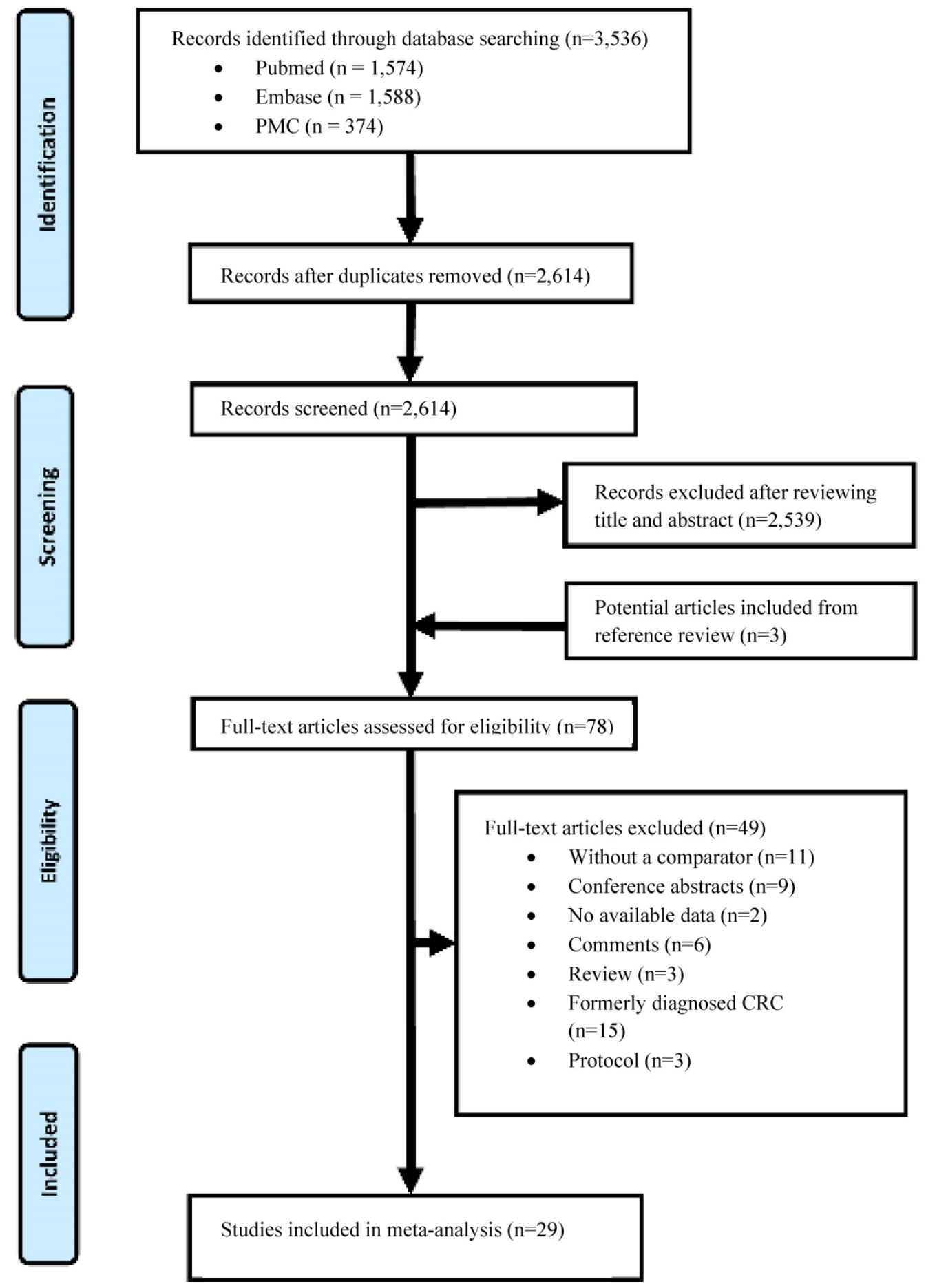

Figure 1. Flow diagram of study selection. 
potentially relevant. After reviewing the titles and abstracts, a total of 75 articles were considered relevant. Three studies were found to be eligible for inclusion in the manual search process, 78 records left. 49 citations were further excluded after carefully reading the full text. The reasons for exclusion were as follows: without a comparator $(n=11)$, conference abstracts $(n=9)$, no available data $(n=2)$, comments $(n=6)$, review $(n=3)$ and formerly diagnosed CRC $(\mathrm{n}=15)$, protocol $(\mathrm{n}=3)$. Finally, twenty-nine articles [8, $10,11,24,25,27,35-57]$ were enrolled for metaanalysis.

\section{Study Characteristics}

NOS scores and detailed characteristics of the 29 records are presented in Supplementary Table 4 \& Table 1, respectively. Among the eligible 29 studies, sixteen were case-control studies $[10,24,25,27,40,42$,
$43,48,50-57]$, while the remaining were cohort studies $[8,11,35-39,41,44-47,49]$. This meta-analysis included $4,713,778$ individuals, three of the studies $[35,38,47]$ included more than 1 million individuals, two studies $[10,57]$ more than 100,000 individuals, ten studies $[8,24,25,36,37,41,44-46,54]$ included 10,000100,000 individuals, and fourteen studies $[11,27,39$, $40,42,43,48-53,55,56]$ enrolled less than 10,000 individuals. Table 2 presented the characteristics of interventions. Among the included studies, there are 19 studies adopted colonoscopic screening, while the remaining study was followed by diagnostic. The regions included in the study were as follows: one from Japan, five from Europe, three from Canada and twenty from the USA. Fourteen and nine studies only reported the incidence or mortality of CRC, respectively, and five reported both.

Table 1. Characteristics and quality of Studies Included in the Meta-analysis

\begin{tabular}{|c|c|c|c|c|c|c|c|c|c|c|}
\hline Study & Year & Design & Country & $\begin{array}{l}\text { Study } \\
\text { period }\end{array}$ & Sample size & Age,Y & $\begin{array}{l}\text { Men } \\
(\%)\end{array}$ & $\begin{array}{l}\text { Follow, } \\
\text { years }\end{array}$ & $\begin{array}{l}\text { Adjustments or } \\
\text { Match }\end{array}$ & $\begin{array}{l}\text { Quality } \\
\text { score }\end{array}$ \\
\hline Ko et al. [57] & 2019 & $\mathrm{CC}$ & USA & $2004-2013$ & $133,279(40,875 / 92,404)$ & $70-85$ & 44.6 & 5 & $1-4,10,15$ & NOS: 7 \\
\hline Lee et al. [35] & 2019 & $\mathrm{RC}$ & USA & 1998-2016 & $1,251,318(991,945 / 259,373)$ & $50-75$ & 49 & 8 & $1-3,7,10$ & NOS: 8 \\
\hline Doubeni et al. [27] & 2018 & $\mathrm{NCC}$ & USA & 2006-2012 & $5207(1747 / 3460)$ & $55-89$ & 49.4 & 10 & $1,2,13,15$ & NOS: 7 \\
\hline Niikura et al. [36] & 2017 & $\mathrm{RC}$ & Asia & 2001-2015 & $85,980(18,816 / 67,119)$ & $>20$ & 68.51 & 6 & 1,2 & NOS: 8 \\
\hline Wang et al. [37] & 2016 & $\mathrm{RC}$ & USA & 1998-2005 & $30,138(5701 / 24,437)$ & $76-85$ & 65 & 4 & $1,2,4,12,14,15$ & NOS: 7 \\
\hline Stock et al. [38] & 2016 & PC & $\begin{array}{l}\text { North } \\
\text { America }\end{array}$ & 1992-2009 & $\begin{array}{l}1,509,423 \\
(177,465 / 1,331,958)\end{array}$ & $60-80$ & 46.06 & 8 & $1,2,9,10$ & NOS: 7 \\
\hline $\begin{array}{l}\text { Ananthakrishnan } \\
\text { et al. [39] }\end{array}$ & 2015 & $\mathrm{RC}$ & USA & NR & $6823(2764 / 4059)$ & $\begin{array}{l}\text { screened } 47(32-61) \\
\text { Never screened } 49 \text { (35-63) }\end{array}$ & 45.5 & 3 & $1-3$ & NOS: 7 \\
\hline Kahi et al. [40] & 2014 & $\mathrm{CC}$ & USA & $1997-2007$ & $2,492(623 / 1,869)$ & $81.22 \pm 3.89$ & 98.7 & 5.19 & $1-3$ & NOS: 7 \\
\hline Morois et al. [41] & 2014 & PC & Europe & $1990-2008$ & $92,048(37,459 / 54,589)$ & $\begin{array}{l}\text { screened: } 49.9 \pm 6.6 \text {; } \\
\text { control: } 48.8 \pm 6.6\end{array}$ & NR & 15.4 & $1,6-8,17$ & NOS: 7 \\
\hline Brenner et al. [42] & 2014 & $\mathrm{CC}$ & Europe & $1993-2010$ & $4,800(2,516 / 2,284)$ & 70 & 59 & 10 & $1,2,6-8,15,17$ & NOS: 7 \\
\hline Doubeni et al. [43] & 2013 & $\mathrm{CC}$ & USA & 2006-2008 & $980(471 / 509)$ & $55-85$ & 51.4 & 5 & $1,2,5,8,9$ & NOS: 7 \\
\hline Wang et al. [44] & 2013 & RC & USA & $1998-2005$ & $53,676(12,266 / 41,410)$ & $\begin{array}{l}\text { screened: } 73.1 \pm 3.8 \text {; } \\
\text { control: } 73.3 \pm 4.0\end{array}$ & 39.3 & 5 & $1-4,15,17$ & NOS: 7 \\
\hline Nishihara et al. [8] & 2013 & PC & USA & 1988-2012 & 88,902 (NA/NA) & Men: 42-77; Women: 32-57 & 35.7 & NR & $1,2,6-9$ & NOS: 7 \\
\hline Eldridge et al. [45] & 2013 & PC & USA & 1995-2008 & $68,531(22,780 / 45,751)$ & $50-71$ & 62 & 11 & $1-3,5,8,17$ & NOS: 7 \\
\hline Manser et al. [46] & 2012 & PC & Europe & $2000-2007$ & $22,686(1912 / 20,774)$ & $50-80$ & 57.8 & 6 & $1,2,5-8,16$ & NOS: 7 \\
\hline Jacob et al. [47] & 2012 & RC & $\begin{array}{l}\text { North } \\
\text { America }\end{array}$ & 1996-2007 & $1,089,998(86,837 / 1,003,161)$ & $50-74$ & 45.1 & 7 & $1,2,4$ & NOS: 6 \\
\hline Baxter et al. [25] & 2012 & $\mathrm{CC}$ & USA & 1991-2007 & $37,099(9,458 / 27,641)$ & $\begin{array}{l}\text { screened: } 79.9 \text { (70.0-89.9) } \\
\text { control: } 79.8(69.1-90.8)\end{array}$ & 42.6 & 9.4 & $1-4,15-17$ & NOS: 7 \\
\hline Schoen et al. [10] & 2012 & $\mathrm{CC}$ & USA & $1993-2001$ & $154,890(77,445 / 77.445)$ & $55-74$ & 49.5 & 3 & $1-3$ & NOS: 7 \\
\hline Mulder et al. [48] & 2010 & $\mathrm{CC}$ & Europe & $1996-2005$ & $8384(594 / 7790)$ & $69.5 \pm 11.9 / 69.3 \pm 11.9$ & 51.7 & 9 & $1,2,11$ & NOS: 6 \\
\hline Kahi et al. [11] & 2009 & $\mathrm{RC}$ & USA & $1989-2007$ & 733 (NA/NA) & $61 \pm 6.5$ & 59 & 2 & 1,2 & NOS: 7 \\
\hline Blom et al. [49] & 2008 & PC & Europe & $1996-2004$ & 1,986 (NA/NA) & $59-61$ & NR & 2 & 1,2 & NOS: 7 \\
\hline Cotterchio et al. [50] & 2005 & $\mathrm{CC}$ & $\begin{array}{l}\text { North } \\
\text { America }\end{array}$ & $1997-2000$ & $2,915(971 / 1,944)$ & $20-74$ & 52 & 2 & $1,2,5-9,15,17$ & NOS: 7 \\
\hline Newcomb et al. [51] & 2003 & $\mathrm{CC}$ & USA & 1998-2002 & $2,962(1,668 / 1,294)$ & $20-74$ & NR & 5 & $1,2,6-8,17$ & NOS: 7 \\
\hline Slattery et al. [52] & 2000 & $\mathrm{CC}$ & USA & NR & $2,893(1,349 / 1,544)$ & $30-67$ & NR & 5 & $1,2,5,7,8$ & NOS: 8 \\
\hline Scheitel et al. [53] & 1999 & $\mathrm{CC}$ & USA & $1970-1993$ & $653(218 / 435)$ & $45-97$ & 42.2 & 10 & $1,2,5,8$ & NOS: 7 \\
\hline Müller et al. [24] & 1995 & $\mathrm{CC}$ & USA & 1978-1992 & $20,889(4,358 / 16,531)$ & $\begin{array}{l}\text { Cases (CC): } 69.1 \\
\text { Cases (RC): } 68.3 \\
\text { Control: } 57.0\end{array}$ & 97.7 & 8.3 & $1-3,8$ & NOS: 7 \\
\hline Müller et al. [54] & 1995 & $\mathrm{CC}$ & USA & 1981-1993 & $32,702(16,351 / 16,351)$ & $\begin{array}{l}\text { Cases (CC): } 67.2 \pm 9.3 \\
\text { Cases (RC): } 66.2 \pm 9.4 \\
\text { Control: } 57.0\end{array}$ & 97.8 & 7 & $1-3$ & NOS: 7 \\
\hline Selby et al. [55] & 1992 & $\mathrm{CC}$ & USA & $1971-1987$ & $1129(261 / 868)$ & $40-50$ & 59.4 & 10 & $1,2,8$ & NOS: 6 \\
\hline Newcomb et al. [56] & 1992 & $\mathrm{CC}$ & USA & 1979-1988 & $262(66 / 196)$ & $50-80$ & NR & 5 & $1,2,5,8$ & NOS: 7 \\
\hline
\end{tabular}


Table 2. Results and meta-analyses of observational studies on the effects of colonoscopy on CRC. Values are relative risks $(95 \%$ confidence intervals) unless stated otherwise

\begin{tabular}{|c|c|c|c|c|}
\hline Study & Year & Intervention & Incidence & Mortality \\
\hline Ko et al. [57] & 2019 & Screening & $0.41(0.39-0.43)$ & NR \\
\hline Lee et al. [35] & 2019 & Screening & $0.54(0.31-0.94)$ & $0.12(0.02-0.82)$ \\
\hline Doubeni et al. [27] & 2018 & Screening & NR & $0.33(0.21-0.52)$ \\
\hline Niikura et al. [36] & 2017 & Various* & $0.50(0.34-0.68)$ & $0.08(0.02-0.17)$ \\
\hline Wang et al. [37] & 2016 & Screening & $0.42(0.28-0.65)$ & NR \\
\hline Stock et al. [38] & 2016 & Screening & NR & $0.36(0.33-0.38)$ \\
\hline $\begin{array}{l}\text { Ananthakrishnan et al. } \\
\text { [39] }\end{array}$ & 2015 & Screening & $0.65(0.45-0.93)$ & $0.34(0.12-0.95)$ \\
\hline Kahi et al. [40] & 2014 & $\begin{array}{l}\text { Screening/ } \\
\text { diagnostic }\end{array}$ & $0.57(0.47-0.70)$ & NR \\
\hline Morois et al. [41] & 2014 & Screening & $0.56(0.47-0.68)$ & NR \\
\hline Brenner et al. [42] & 2014 & Screening & $0.11(0.08-0.15)$ & NR \\
\hline Doubeni et al. [43] & 2013 & Various* & $0.29(0.15-0.58)$ & NR \\
\hline Wang et al. [44] & 2013 & $\begin{array}{l}\text { Screening/ } \\
\text { diagnostic }\end{array}$ & $0.34(0.25-0.46)$ & NR \\
\hline Nishihara et al. [8] & 2013 & Screening & NR & $0.32(0.24-0.45)$ \\
\hline Eldridge et al. [45] & 2013 & Screening & NR & $0.41(0.30-0.55)$ \\
\hline Manser et al. [46] & 2012 & Screening & $0.31(0.16-0.59)$ & $0.12(0.01-0.93)$ \\
\hline Jacob et al. [47] & 2012 & $\begin{array}{l}\text { Screening/ } \\
\text { diagnostic }\end{array}$ & $0.52(0.34-0.76)$ & $0.19(0.07-0.47)$ \\
\hline Baxter et al. [25] & 2012 & $\begin{array}{l}\text { Screening/ } \\
\text { diagnostic }\end{array}$ & NR & $0.40(0.37-0.43)$ \\
\hline Schoen et al. [10] & 2012 & Screening & $0.79(0.72-0.85)$ & NR \\
\hline Mulder et al. [48] & 2010 & diagnostic & $0.56(0.33-0.94)$ & NR \\
\hline Kahi et al. [11] & 2009 & Screening & $0.52(0.22-0.82)$ & NR \\
\hline Blom et al. [49] & 2008 & Screening & $0.50(0.20-1.30)$ & NR \\
\hline Cotterchio et al. [50] & 2005 & Various* & $0.52(0.34-0.80)$ & NR \\
\hline Newcomb et al. [51] & 2003 & Screening & $0.24(0.17-0.33)$ & NR \\
\hline Slattery et al. [52] & 2000 & Screening & NR & NR \\
\hline Scheitel et al. [53] & 1999 & Screening & NR & $0.89(0.47-1.66)$ \\
\hline Müller et al. [24] & 1995 & diagnostic & NR & $0.45(0.30-0.66)$ \\
\hline Müller et al. [54] & 1995 & diagnostic & $0.53(0.41-0.69)$ & NR \\
\hline Selby et al. [55] & 1992 & Screening & NR & $0.41(0.25-0.69)$ \\
\hline Newcomb et al. [56] & 1992 & Screening & NR & $0.21(0.08-0.52)$ \\
\hline
\end{tabular}

\section{Quality of included studies}

Quality assessment was shown in Supplementary Table 4. Among these 29 eligible studies, the scores of Newcastle-Ottawa quality were ranging from 6 to 8 . All studies scored six stars or more. Moreover, most studies were adjusted or matched for the following confounders: age (29/29), sex (29/29) (Table 1).

\section{Incidence reduction of CRC by colonoscopy}

As for incidence, a total of 19 studies were calculated the combination of $R R$ and $95 \% C I$ within a fixed-effects model and the values were pooled $R R=$ $0.48,95 \% C I=0.46-0.49$, indicating that colonoscopy can reduce the CRC mortality of 52\% RR (Figure 2A). However, there was high heterogeneity among studies $\left(I^{2}=94.0 \%, p=0.000\right)$. To explore the source of heterogeneity, sensitivity analysis was carried out (Figure 2B), indicating that the studies of $\mathrm{Ko}$ et al, Brenner et al., and Schoen et al., [10,42,57] had a great impact on the pooled $R R$. Hence, these three articles were excluded and meanwhile, the incidence rate decreased slightly $(R R, 0.49 ; 95 \% C I, 0.45-0.53)$, as well as the heterogeneity decreased ( $I^{2}=57.7 \%, p=$ 0.002) (Figure 2C). Sensitivity analysis showed that none of these 16 studies could have a great impact on the pooled $R R$ (Figure 2D).

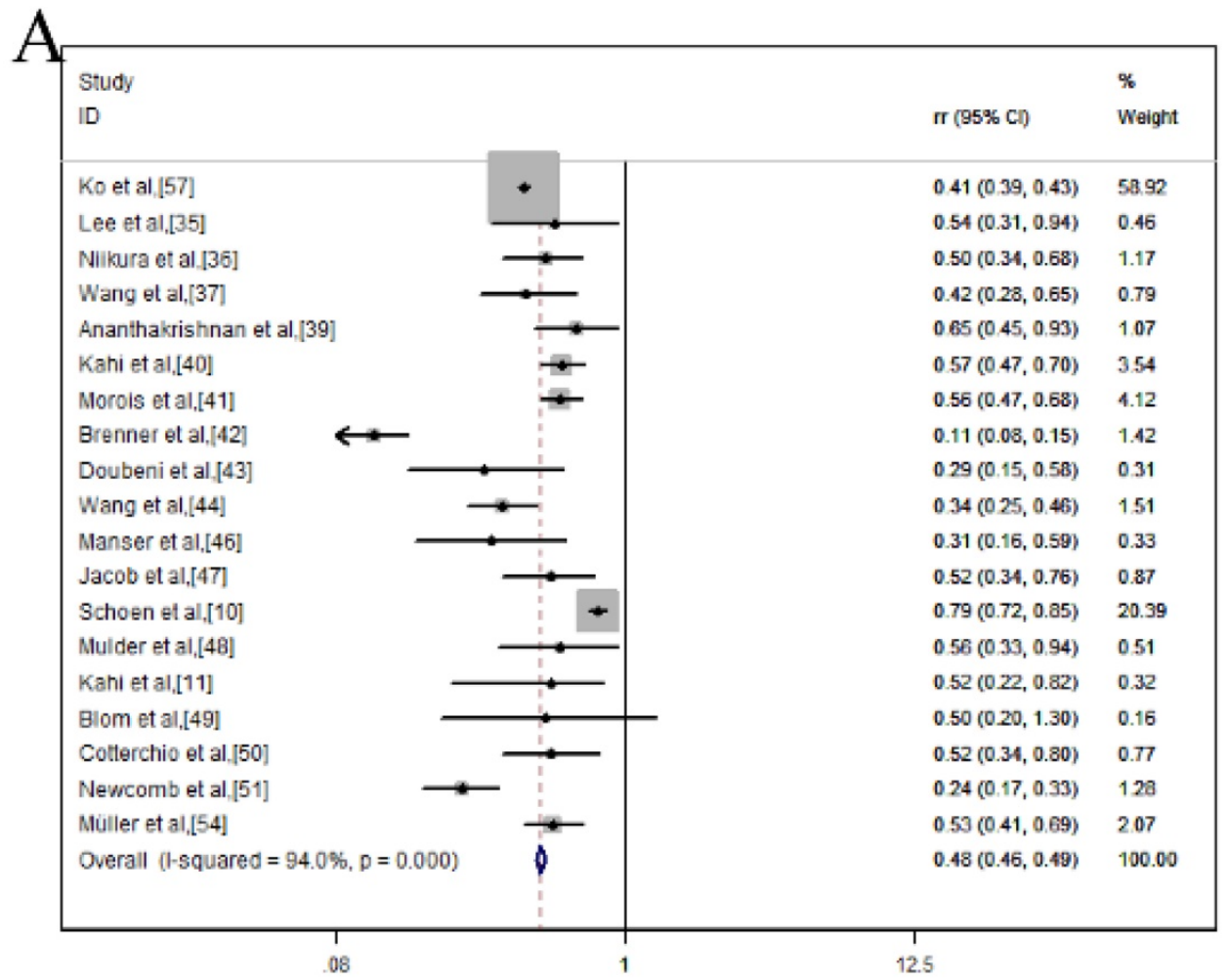


B

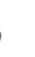

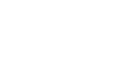

\begin{tabular}{|c|c|c|}
\hline Study & & $\mathbf{s}$ \\
\hline ID & $n(95 \% \mathrm{Cl})$ & Weight \\
\hline Lee et al,[35] & $0.54(0.31,0.94)$ & 2.37 \\
\hline Niikura et al,[36] & $0.50(0.34,0.08)$ & 0.07 \\
\hline Wang et al,[37] & $0.42(0.28,0.60)$ & 4.11 \\
\hline Ananthakrishnan et al,[39] & $0.06(0.45,0.93)$ & 0.63 \\
\hline Kahi et al,[40] & $0.57(0.47,0.70)$ & 18.37 \\
\hline Morois et al [41] & $0.58(0.47 .0 .88)$ & 21.37 \\
\hline Doubeni et al, [43] & $0.29(0.15 .0 .58)$ & 159 \\
\hline Wang et al,[44] & $0.34(0.25,0.46)$ & 784 \\
\hline Manser et al, [46] & $0.31(0.10,0.59)$ & 1.71 \\
\hline Jacob et al,[47] & $0.52(0.34,0.70)$ & 4.51 \\
\hline Mulder et al.[48] & $0.50(0.33,0.94)$ & 2.00 \\
\hline Kahi et al,[11] & $0.02(0.22,0.82)$ & 1.08 \\
\hline Blom et al,[49] & $0.00(0.20,1.30)$ & 0.83 \\
\hline Catterchio et al, [50] & $0.52(0.34,0.80)$ & 3.90 \\
\hline Newcomb et al, [51] & $0.24(0.17 .0 .33)$ & 6.63 \\
\hline Müller et al, \{54] & $0.53(0.41,0.09)$ & 10.70 \\
\hline Overall (l-squared $=57.7 \%, p=0.002)$ & $0.49(0.45,0.53)$ & 100.00 \\
\hline
\end{tabular}

D

Meta-analysis estimates, given named study is omitted

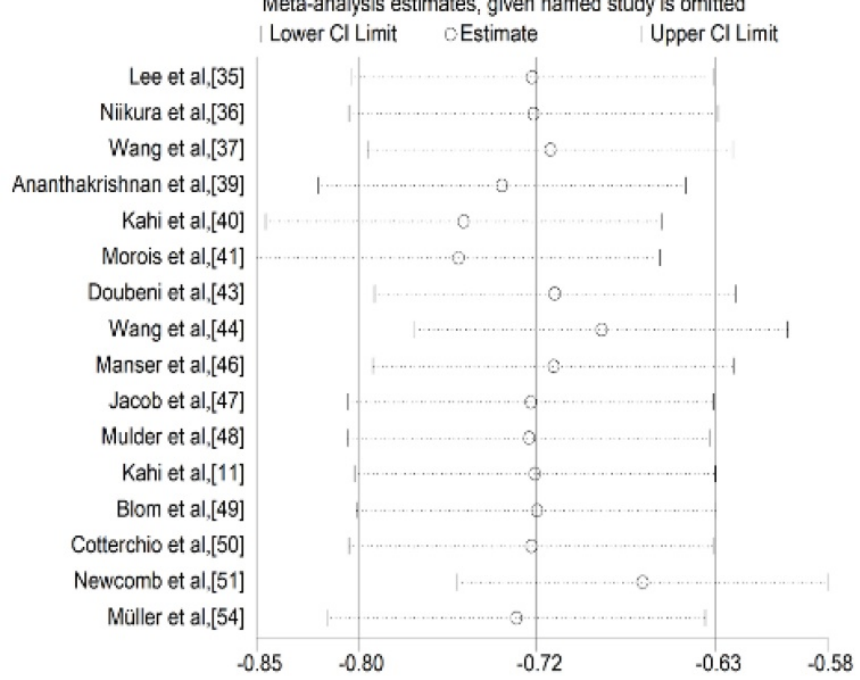

Figure 2. Colonoscopy associated with the incidence reduction of CRC and after excluding one related article; (A) Forest plot; (B) Sensitivity analysis; (C) Forest plot; (D) Sensitivity analysis. 
Table 3. Subgroup analysis of CRC incidence reduction after endoscopic screening

\begin{tabular}{|c|c|c|c|c|c|c|}
\hline \multirow[t]{2}{*}{ Subgroups } & \multirow[t]{2}{*}{ No. of studies } & \multirow[t]{2}{*}{ Pooled RR (95\% CI) } & \multirow[t]{2}{*}{$Z$} & \multirow[t]{2}{*}{$P$} & \multicolumn{2}{|c|}{ Heterogeneity } \\
\hline & & & & & $\mathrm{I} 2(\%)$ & $\mathrm{Ph}$ \\
\hline Intervention & & & & & & 0.29 \\
\hline Screening $[11,35,37,39,41,46,49,51]$ & 8 & $0.475(0.418-0.540)$ & 5.57 & 0.000 & 71.4 & \\
\hline Screening/diagnostic and Various* $[36,40,43,44,47,48,50,54]$ & 8 & $0.498(0.444-0.558)$ & 9.28 & 0.000 & 34.8 & \\
\hline Study design & & & & & & 0.28 \\
\hline Cochort $[11,35-37,39,41,44,46,47,49]$ & 10 & $0.498(0.444-0.558)$ & 9.52 & 0.000 & 27.6 & \\
\hline (Nested) case-control $[40,43,48,50,51,54]$ & 6 & $0.475(0.418-0.540)$ & 5.28 & 0.000 & 78.0 & \\
\hline Country & & & & & & 0.02 \\
\hline Western $[11,35,37,39-41,43,44,46-51,54]$ & 15 & $0.487(0.446-0.532)$ & 9.62 & 0.000 & 60.5 & \\
\hline Asia [36] & 1 & $0.500(0.354-0.707)$ & 3.92 & 0.000 & NA & \\
\hline Sample size & & & & & & 0.24 \\
\hline$\leq 10,000[11,39,40,43,48-51]$ & 8 & $0.484(0.424-0.553)$ & 5.23 & 0.000 & 72.0 & \\
\hline $10,000-100,000[36,37,41,44,46,54]$ & 6 & $0.486(0.431-0.547)$ & 8.14 & 0.000 & 51.3 & \\
\hline$\geq 1$ million $[35,47]$ & 2 & $0.527(0.380-0.730)$ & 3.86 & 0.000 & 0 & \\
\hline Age & & & & & & 0.01 \\
\hline $20-50[36,39,41,50,51]$ & 5 & $0.491(0.431-0.558)$ & 4.46 & 0.000 & 82.0 & \\
\hline$\geq 50[11,35,37,40,43,44,46-49,54]$ & 11 & $0.485(0.433-0.544)$ & 10.23 & 0.000 & 24.6 & \\
\hline Sex & & & & & & 9.04 \\
\hline Male $[36,40,47,48,52]$ & 5 & $0.473(0.390-0.573)$ & 7.64 & 0.000 & 0.0 & \\
\hline Female $[36,37,47,48,52]$ & 5 & $0.702(0.592-0.833)$ & 3.40 & 0.001 & 29.6 & \\
\hline
\end{tabular}

$\mathrm{NA}=$ not applicable; $\mathrm{RR}=$ relative risk $\mathrm{CI}=$ confidence interval .

\section{Subgroup analysis of CRC incidence reduction}

As presented in Table 3 and Figure 3, we conducted a subgroup analysis of CRC incidence reduction after endoscopic screening based on different interventions, study design, country, sample size, age and sex. We found that colonoscopy could significantly reduce the CRC incidence compared with never-screened $(R R=0.475 ; 95 \% C I=0.418$ $0.540 ; p \leq 0.001 ; I^{2}=71.4 \%$ ) (Figure 3A). In the subgroup analysis of the study design, colonoscopy provided protection in both cohort $(R R=0.498 ; 95 \% C I$ $\left.=0.444-0.558 ; p \leq 0.001 ; I^{2}=27.6 \%\right)$ and case-control studies $\left(R R=0.475 ; 95 \% C I=0.418-0.540 ; p \leq 0.001 ; I^{2}\right.$ $=78.0 \%$ ) (Figure 3B). In the region-based grouping analysis, the incidence of CRC decreased in both western $\left(R R=0.487,95 \% C I=0.446-0.532 ; p \leq 0.001 ; I^{2}\right.$ $=60.5 \%)$ and eastern $(R R=0.500,95 \% C I=0.354-$ $0.707 ; p \leq 0.001$ ) (Figure 3C). Judging from the results of the sample size, colonoscopy can reduce mortality in $\geq 1$ million $(R R=0.527,95 \% C I=0.380-0.730 ; p \leq$ $\left.0.001 ; I^{2}=0.0 \%\right) ; 10,000-100,000(R R=0.486,95 \% C I=$ $\left.0.431-0.547 ; p \leq 0.001 ; I^{2}=51.3 \%\right)$ and less than 10,000 $\left(R R=0.484,95 \% C I=0.424-0.553 ; p \leq 0.001 ; I^{2}=72.0 \%\right)$ (Figure 3D). From the age group, colonoscopy screening provided protection in both 20-50 $(R R=$ $\left.0.491,95 \% C I=0.431-0.558 ; p \leq 0.001 ; I^{2}=82.0 \%\right)$ and $\geq 50\left(R R=0.485,95 \% C I=0.433-0.544 ; p \leq 0.001 ; I^{2}=\right.$ $24.6 \%$ ) (Figure 3E). Similar results were also shown in sex groups (male: $R R=0.473,95 \% C I=0.390-0.573 ; p \leq$ $0.001 ; I^{2}=0.0 \%$; female: $R R=0.702,95 \% C I=$ 0.592-0.833; $p \leq 0.001 ; I^{2}=29.6 \%$ ) (Figure 3F).

\section{Mortality reduction of CRC by colonoscopy}

A total of 14 studies reported a $62 \% R R$ reduction in CRC morality after the colonoscopic screening within a fixed-effects model. The pooled $R R$ was 0.38 $(95 \% C I=0.36-0.40)$ and the heterogeneity was moderate $\left(I^{2}=53.1 \%, p=0.010\right)$ (Figure 4A). To assess whether anyone study had a dominant effect on the meta-analysis RR, each study was excluded, and we found no study markedly affected the summary estimate or P-value for heterogeneity among the other summary estimates (Figure 4B).

\section{Subgroup analysis of CRC mortality reduction}

As displayed in Table 4 and Figure 5, we conducted a subgroup analysis of CRC mortality reduction after endoscopic screening based on different interventions, country, sample size, age and sex. We found that colonoscopy screening had a more significant protective effect than never-screened $(R R=$ $\left.0.362 ; 95 \% C I=0.339-0.386 ; p \leq 0.001 ; I^{2}=31.0 \%\right)$ (Figure 5A). In the subgroup analysis of the study design, colonoscopy provided protection in both cohort $\left(R R=0.356 ; 95 \% C I=0.333-0.381 ; p \leq 0.001 ; I^{2}=\right.$ $45.0 \%)$ and case-control studies $(R R=0.402 ; 95 \% C I=$ $0.375-0.432 ; p \leq 0.001 ; I^{2}=44.5 \%$ ) (Figure 5B). In terms of country, colonoscopy screening provided protection in both western country $(R R=0.378,95 \%$ $\left.C I=0.360-0.397 ; p \leq 0.001 ; I^{2}=38.9 \%\right)$ and eastern country $(R R=0.080 ; 95 \% C I=0.027-0.233 ; p \leq 0.001)$ (Figure 5C). Judging from the results of the sample size, colonoscopy can reduce mortality in these three groups ( $\geq 1$ million: $R R=0.358,95 \% C I=0.334-0.384, p$ $\left.\leq 0.001, I^{2}=34.5 \%\right) ; 10,000-100,000: R R=0.394,95 \% C I$ $=0.368-0.423, p \leq 0.001, I^{2}=58.1 \% ; \leq 10,000: R R=$ $\left.0.409,95 \% C I=0.311-0.537, p \leq 0.001 ; I^{2}=54.4 \%\right)$ ) (Figure 5D). Similar results were also shown in the age (20-50: $R R=0.358,95 \% C I=0.284-0.451, p \leq 0.001$, $I^{2}=71.7 \% ; \geq 50: R R=0.378,95 \% C I=0.360-0.397, p=$ $0.001, I^{2}=29.2 \%$ ) (Figure $5 \mathrm{E}$ ) and sex groups (male: 
$R R=0.440,95 \% C I=0.404-0.479 ; p \leq 0.001 ; I^{2}=49.1 \%$;

$88.5 \%)$ (Figure 5F).

female: $R R=0.351,95 \% C I=0.318-0.388, p \leq 0.001, I^{2}=$

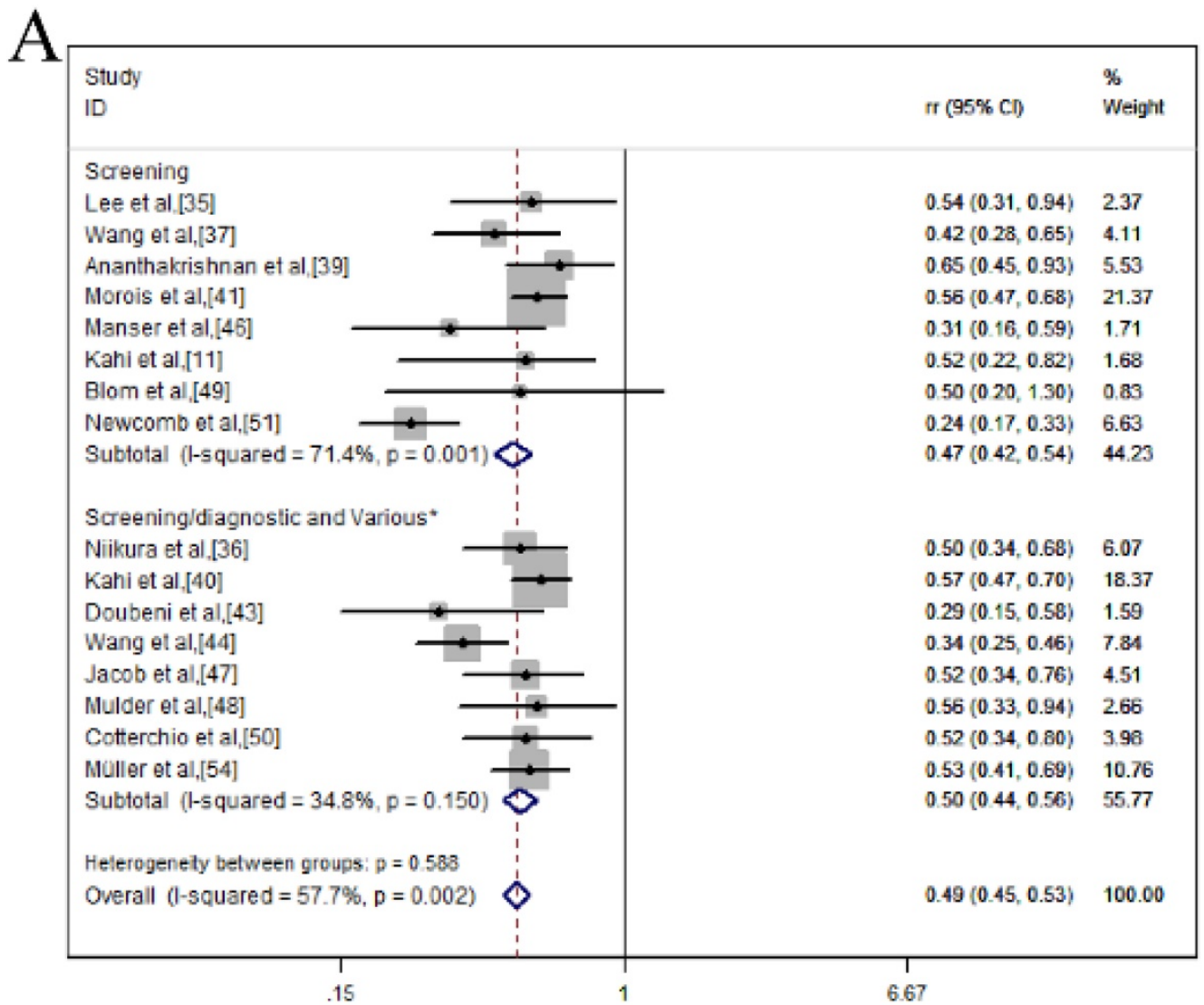

B

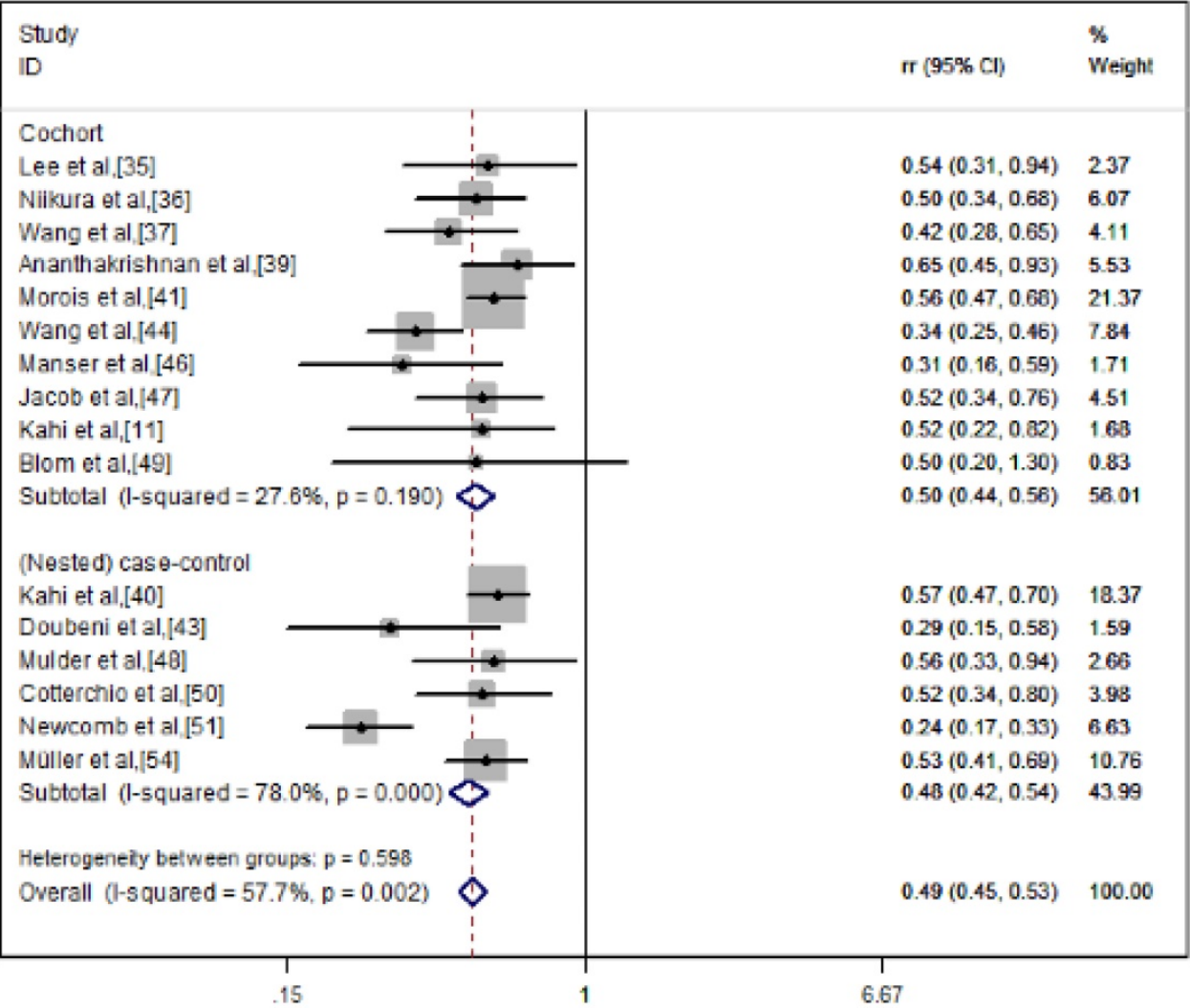


C

$\begin{array}{lll}\text { Study } & & \% \\ \text { ID } & \pi(95 \% \text { Cl) } & \text { Weight }\end{array}$

Western

Lee ot al, [35]

Wang et al,[37]

Ananthakrishnan et al,[39]

Kahi et al [40]

Morois et al,[41]

Doubeni et al, [43]

Wang et al, [44]

Manser et al, [46]

Jacob et al,[47]

Mulder et al,[48]

Kahi et al.[11]

Blom et al.[49]

Cotterchio et al,[50]

Newcomb et al,[51]

Müller et al [54]

Subtotal (l-squared $=60.5 \%, p=0.001)$

Asia

Nilkura et al. [36]

Subtotal (l-squared $=. \%, p=$.)

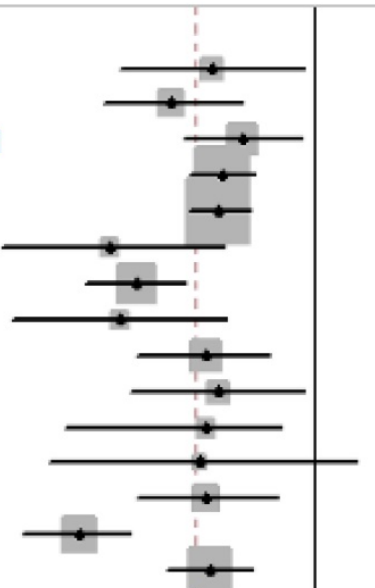

$0.54(0.31,0.94) \quad 2.37$

$0.42(0.28,0.65) \quad 4.11$

$0.65(0.45,0.93) \quad 5.53$

$0.57(0.47,0.70) \quad 18.37$

$0.56(0.47,0.68) \quad 21.37$

$0.29(0.15,0.58) \quad 1.59$

$0.34(0.25,0.46) \quad 7.84$

$0.31(0.16,0.59) \quad 1.71$

$0.52(0.34,0.76) \quad 4.51$

$0.56(0.33,0.94) \quad 2.66$

$0.52(0.22,0.82) \quad 1.68$

$0.50(0.20,1.30) \quad 0.83$

$0.52(0.34,0.80) \quad 3.98$

$0.24(0.17,0.33) \quad 6.63$

$0.53(0.41,0.69) \quad 10.76$

$0.49(0.45,0.53) \quad 93.93$

Heterogeneity between groups: $p=0.884$

Overall (l-squared $=57.7 \%, p=0.002)$

$0.50(0.34,0.68) \quad 6.07$

$0.50(0.35,0.71) \quad 6.07$

$0.49(0.45,0.53) \quad 100.00$

$\begin{array}{lll}15 & & \\ 1 & 6.67\end{array}$

D

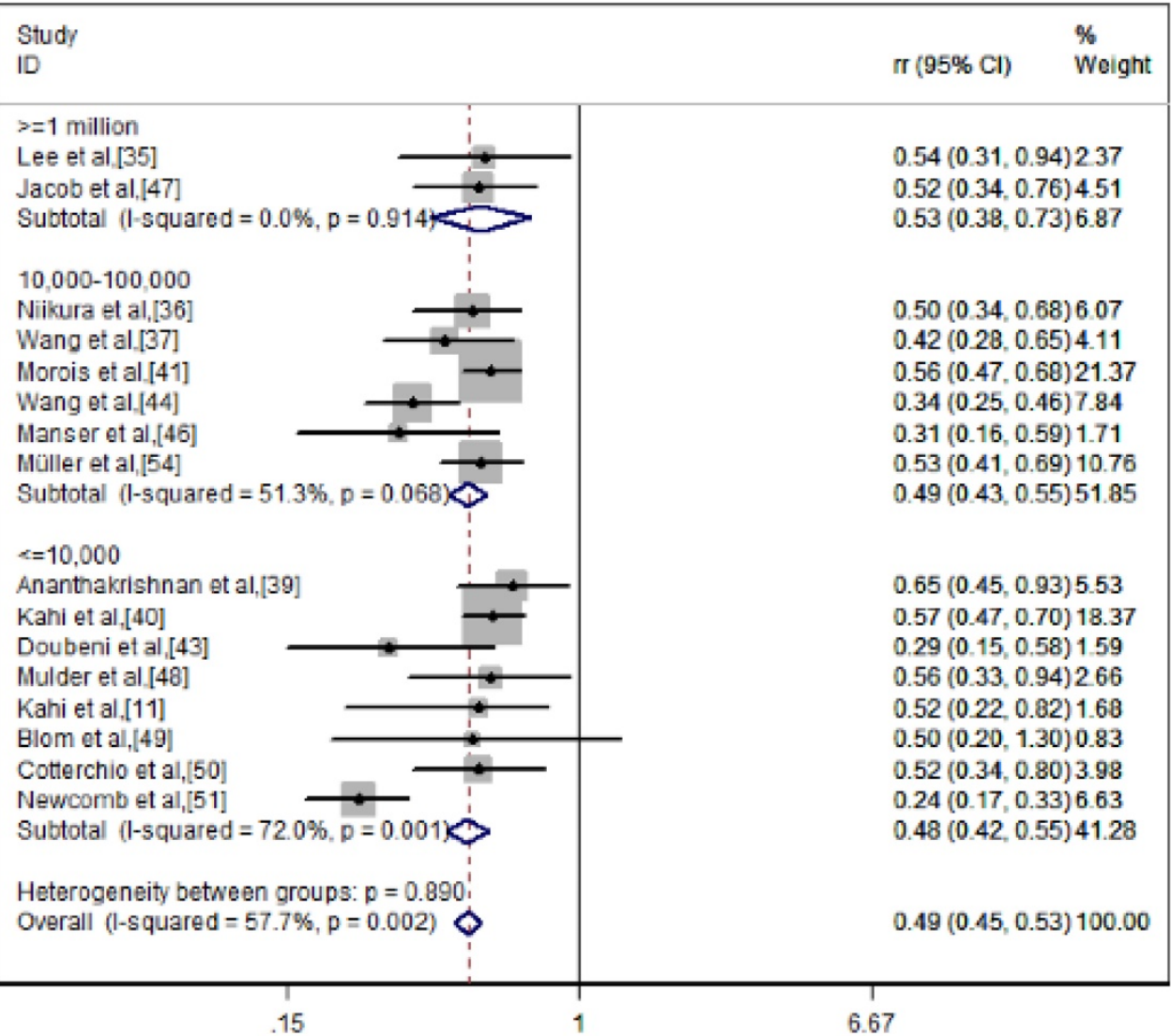


$\mathrm{E}$

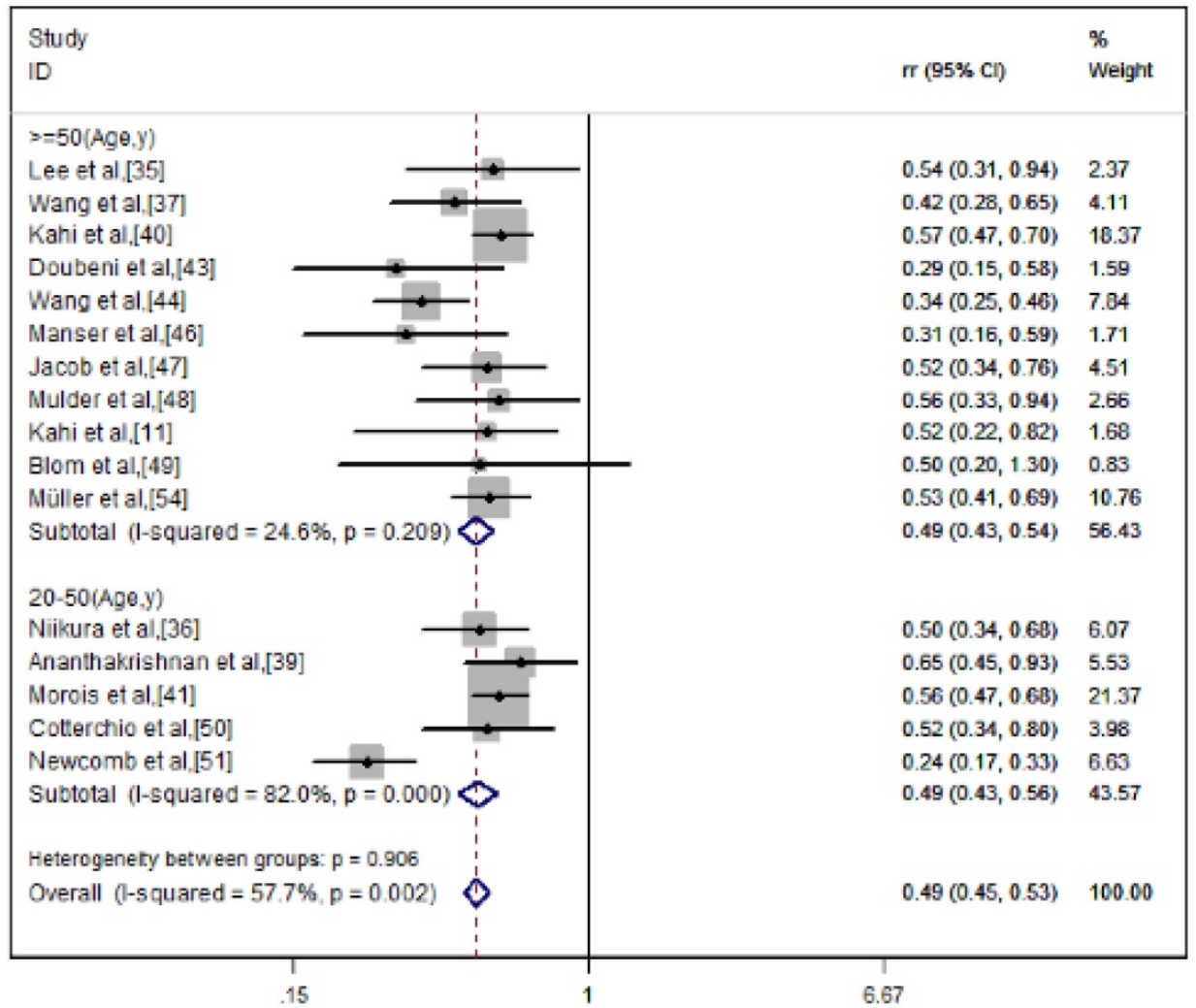

-

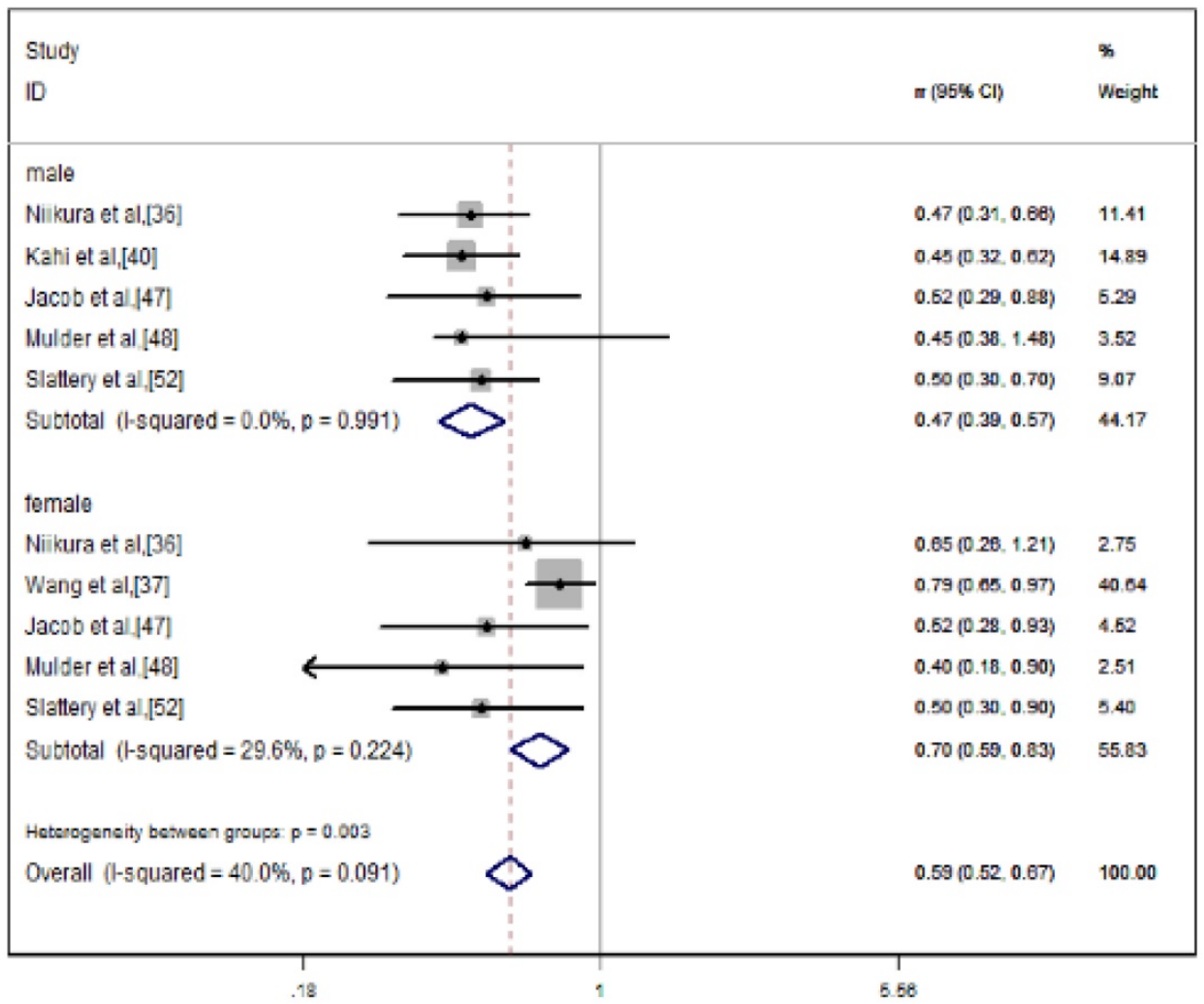

Figure 3. Colonoscopy was associated with a reduced incidence of CRC in a subgroup analysis of forest plots; (A) Intervention; (B) Study design; (C) Country; (D) Sample size; (E) Age; (F) Sex. 


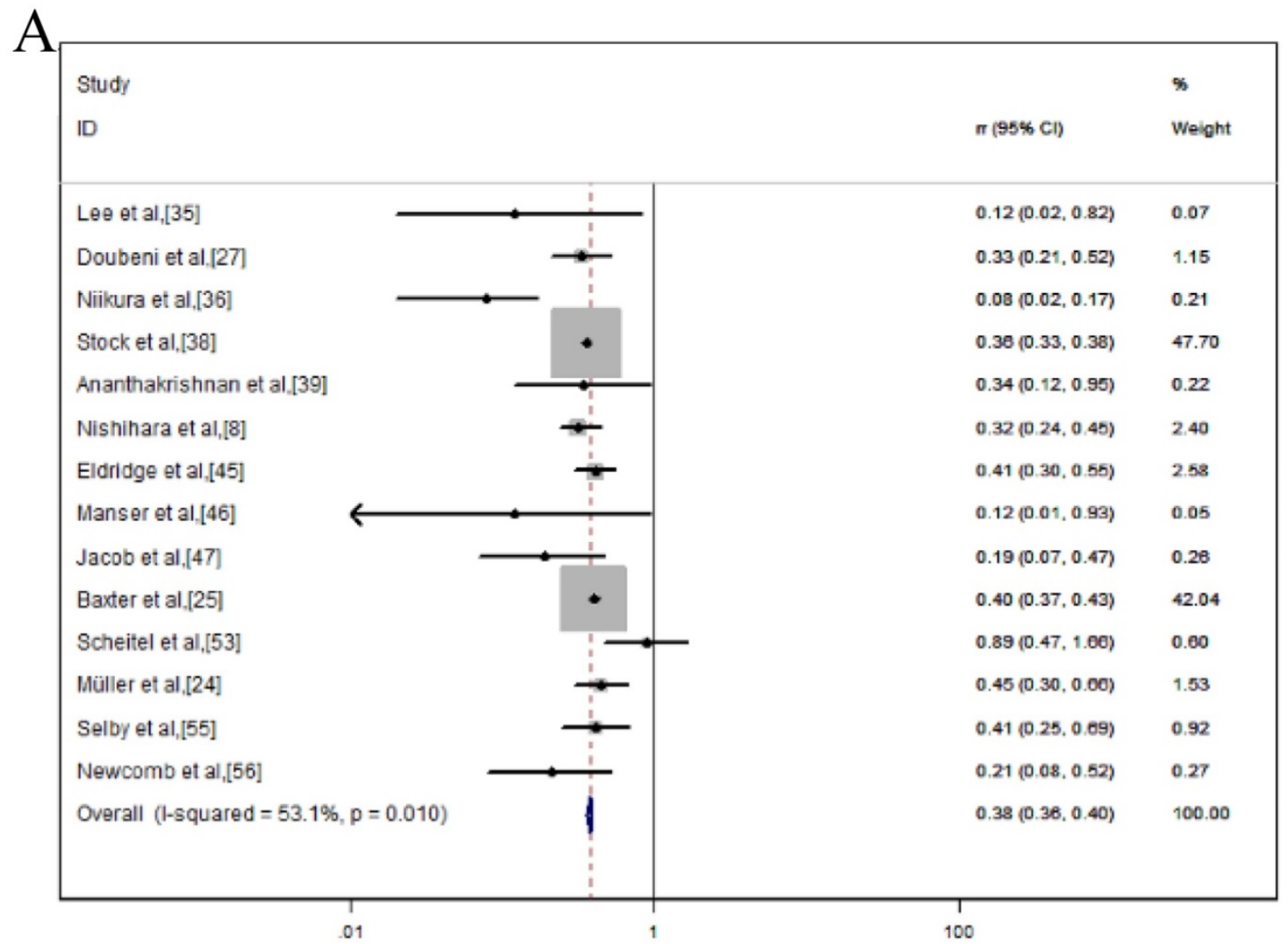

$\mathrm{B}$

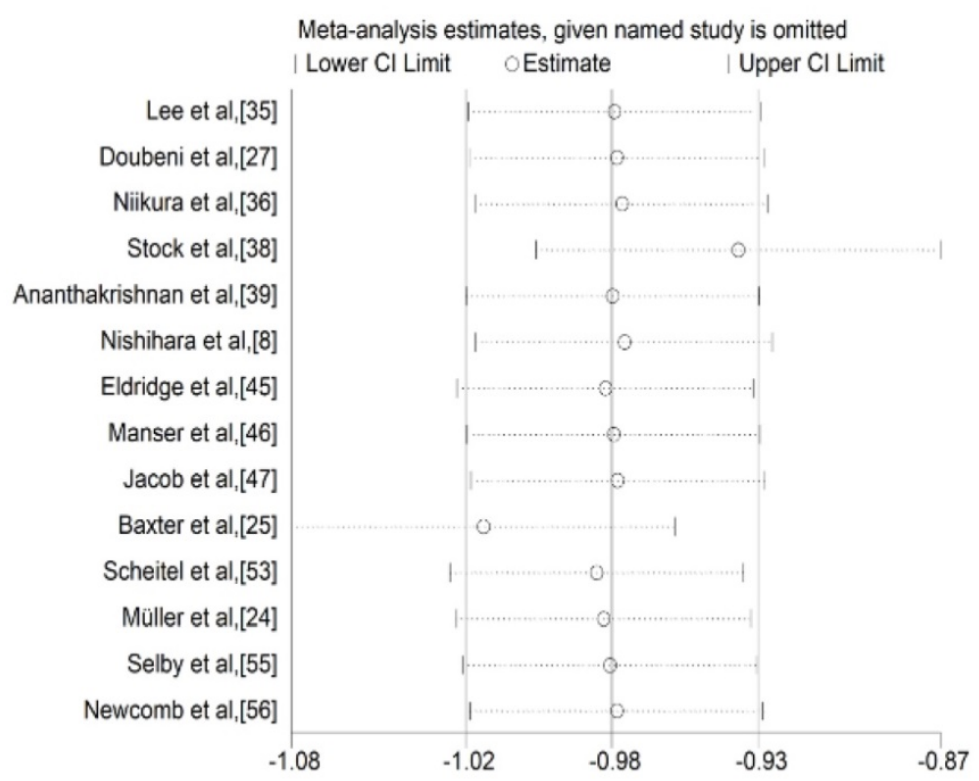

Figure 4. Colonoscopy associated with the mortality reduction of CRC; (A) Forest plot; (B) Sensitivity analysis.

\section{Publication bias}

As displayed in Figure 6, Begg's test combined with Egger's test was utilized to evaluate the publication bias. In the pooled analysis of CRC incidence or mortality reduction after endoscopic screening, the $p$ values of Begg's test and the $p$ values of Egger's test were all above 0.05, indicating that there was no obvious bias among these studies.

\section{Discussion}

This meta-analysis set out with the aim of assessing the importance of colonoscopic screening in preventing CRC incidence and related mortality. Of 
all the 29 studies involving 4,713,778 individuals, our study found a link between colonoscopy and the mortality and incidence of CRC. The outcomes revealed that patients might benefit from $62 \% R R$ and
$52 \% R R$ reduction in CRC mortality $(R R=0.38,95 \% C I$ $=0.36-0.40)$ and incidence $(R R=0.48,95 \% C I=$ 0.46-0.49) after colonoscopic inspection.

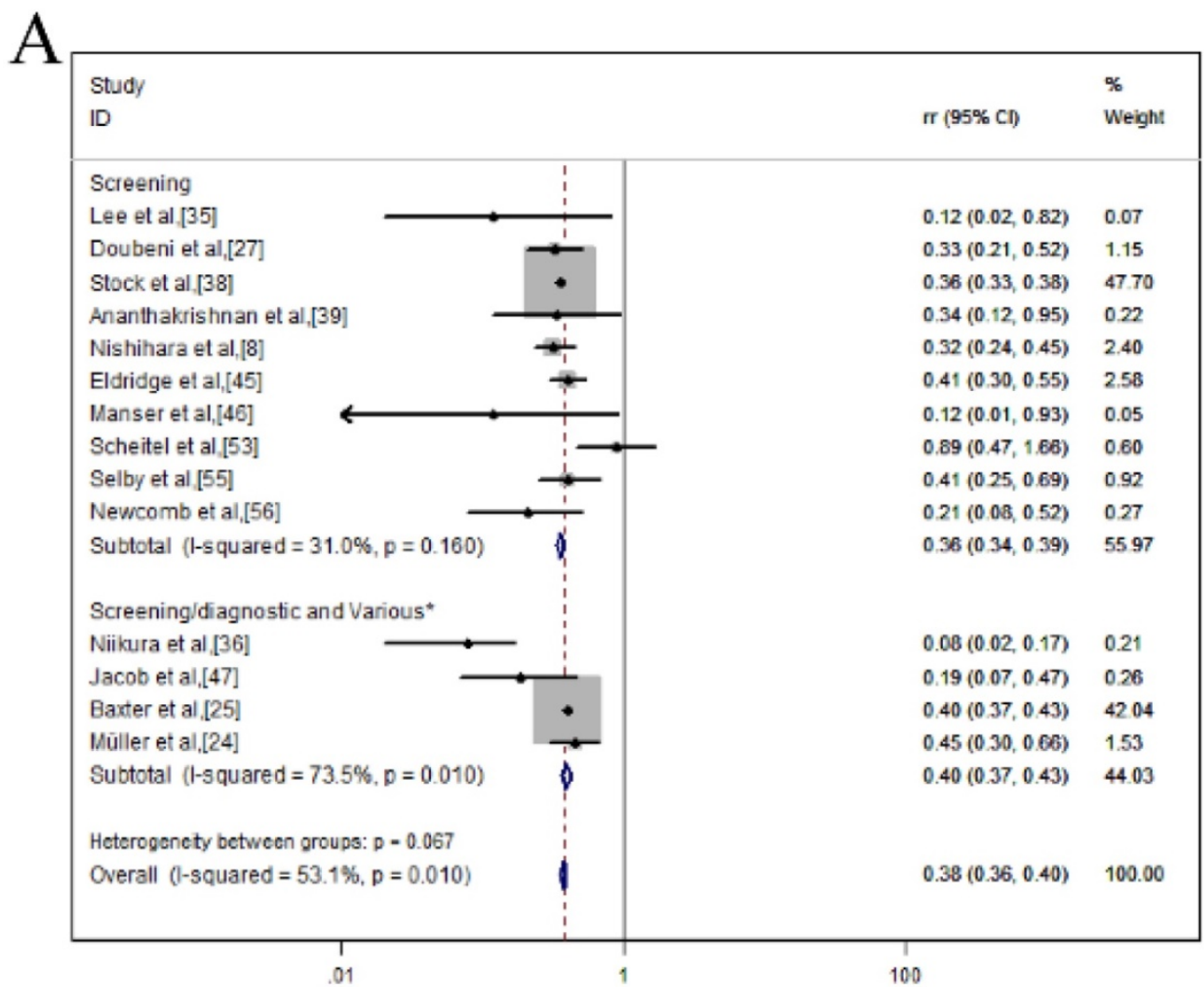

B

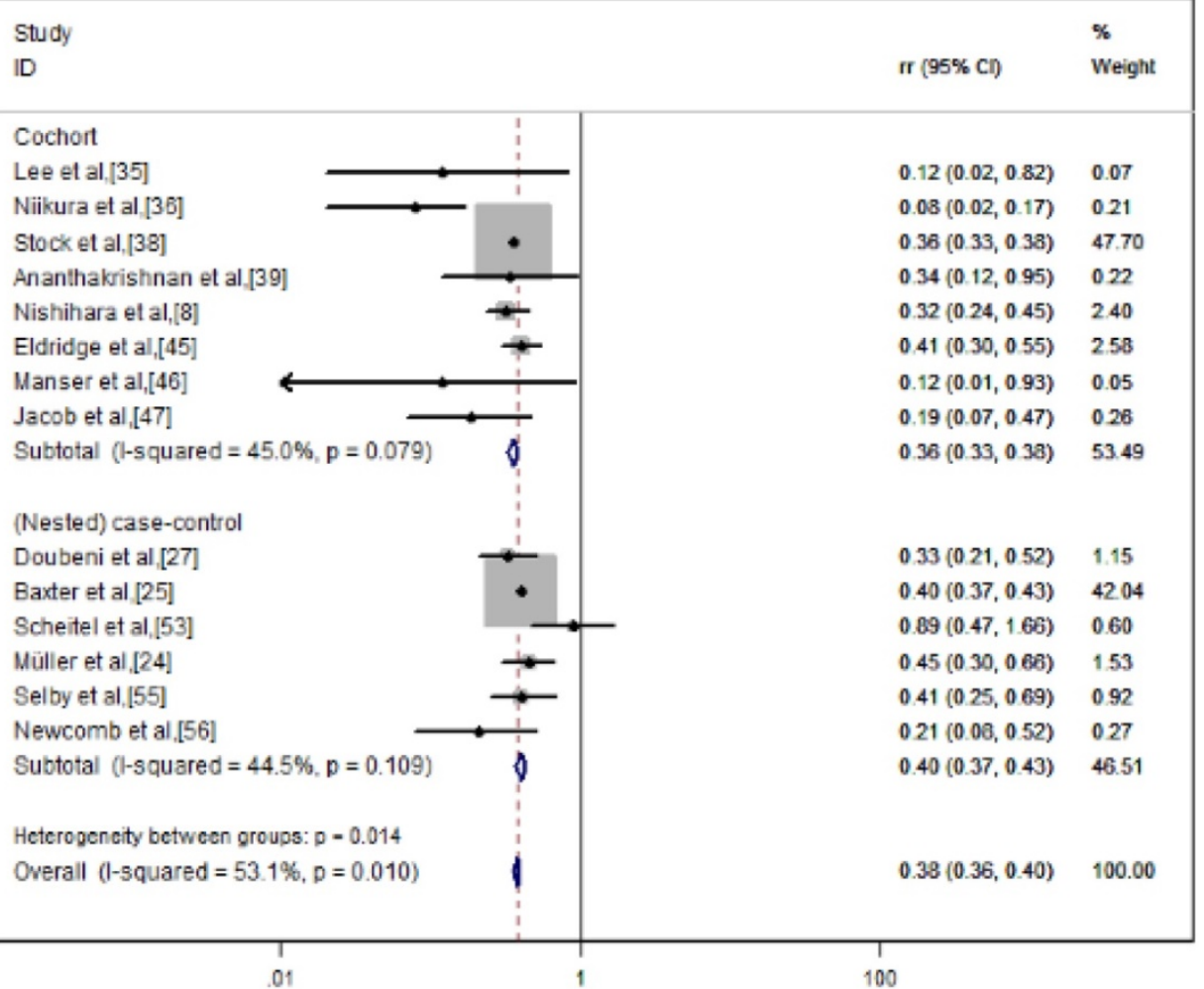


C

\begin{tabular}{|c|c|c|c|}
\hline \multicolumn{2}{|l|}{ Studj } & \multirow[b]{2}{*}{$\pi(95 \% \mathrm{Cl})$} & $\%$ \\
\hline ID & & & Weight \\
\hline \multicolumn{4}{|l|}{ Western } \\
\hline Lee et al,[35] & & $0.12(0.02,0.82)$ & 0.07 \\
\hline Doubeni et al [27] & $\rightarrow$ & $0.33(0.21,0.52)$ & 1.15 \\
\hline Stock et al,[38] & - & $0.36(0.33,0.38)$ & 47.70 \\
\hline Ananthakrishnan et al,[39] & & $0.34(0.12,0.95)$ & 0.22 \\
\hline Nishihara et al,[8] & $\mp$ & $0.32(0.24,0.45)$ & 240 \\
\hline Eldridge et al, [45] & + & $0.41(0.30,0.55)$ & 258 \\
\hline Manser et al,[46] & & $0.12(0.01,0.83)$ & 0.06 \\
\hline Jacob et al,[47] & - & $0.19(0.07,0.47)$ & 0.26 \\
\hline Baxter et al, [25] & - & $0.40(0.37,0.43)$ & 42.04 \\
\hline Scheitel et al,[53] & & $0.89(0.47,1.68)$ & 0.60 \\
\hline Müller et al,[24] & $\rightarrow$ & $0.45(0.30,0.00)$ & 1.53 \\
\hline Selby et al,[55] & $\longrightarrow$ & $0.41(0.25,0.69)$ & 0.92 \\
\hline Newcomb et al,[56] & - & $0.21(0.08,0.52)$ & 0.27 \\
\hline Subtotal (1-squared $=38.9 \%, p=0.074$ ) & 1 & $0.38(0.30 .0 .40)$ & 99.79 \\
\hline \multicolumn{4}{|l|}{ Asia } \\
\hline Nilikura et al_[36] & & $0.08(0.02,0.17)$ & 0.21 \\
\hline Subtotal (l-squared $=. \%, p=)$. & 7 & $0.08(0.03,0.23)$ & 0.21 \\
\hline \multicolumn{4}{|l|}{ Hoterogensity boswoen groups: $p=0.004$} \\
\hline Overall $(1-$ squared $=53.1 \%, p=0.010)$ & 1 & $0.30(0.36,0.40)$ & 100.00 \\
\hline
\end{tabular}

D

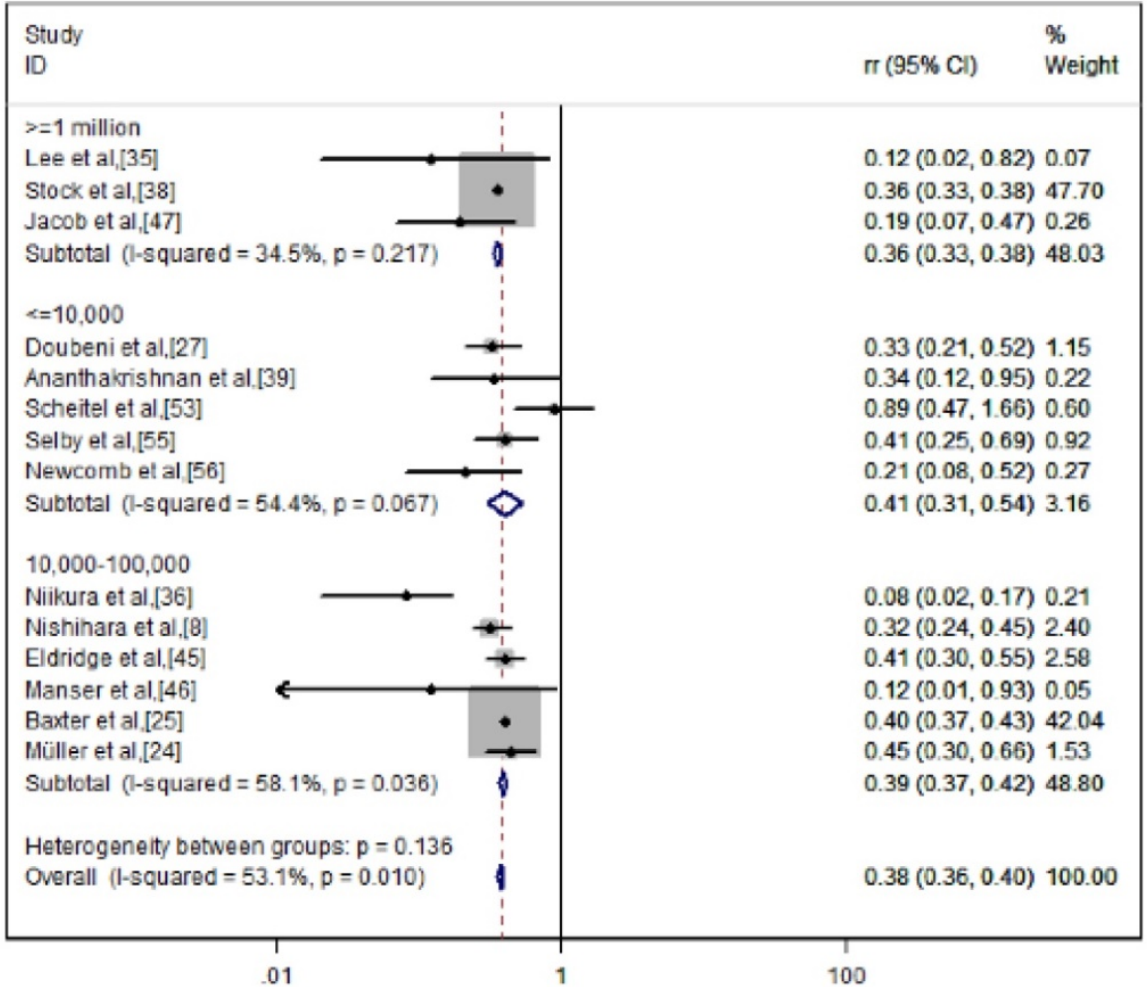


$\mathrm{E}$

\begin{tabular}{|c|c|c|}
\hline \multicolumn{2}{|l|}{ Study } & $\%$ \\
\hline ID & $\pi(95 \%$ Cl) & Weight \\
\hline \multicolumn{3}{|l|}{ 20-50(Age,y) } \\
\hline Lee et al. [35] & $0.12(0.02,0.82)$ & 0.07 \\
\hline Nilkura et al,[36] & $0.08(0.02,0.17)$ & 0.21 \\
\hline Ananthakrishnan et al,[39] & $0.34(0.12,0.95)$ & 0.22 \\
\hline Nishihara et al,[8] & $0.32(0.24,0.45)$ & 2.40 \\
\hline Scheitel et al.,[53] & $0.89(0.47,1.66)$ & 0.60 \\
\hline Selby et al $[55]$ & $0.41(0.25,0.69)$ & 0.92 \\
\hline Subtotal (1-squared $=71.7 \%, p=0.003$ ) & $0.36(0.28,0.45)$ & 4.42 \\
\hline \multicolumn{3}{|l|}{$>=50($ Age,$y)$} \\
\hline Doubeni et al,[27] & $0.33(0.21,0.52)$ & 1.15 \\
\hline Stock et al [38] & $0.36(0.33,0.38)$ & 47.70 \\
\hline Eldridge et al,[45] & $0.41(0.30,0.55)$ & 2.58 \\
\hline Manser et al,[46] & $0.12(0.01,0.93)$ & 0.05 \\
\hline Jacob et al,[47] & $0.19(0.07,0.47)$ & 0.26 \\
\hline Baxter et al,[25] & $0.40(0.37,0.43)$ & 42.04 \\
\hline Mūller et al,[24] & $0.45(0.30,0.66)$ & 1.53 \\
\hline Newcomb et al,[56] & $0.21(0.08,0.52)$ & 0.27 \\
\hline Subtotal (l-squared $=29.2 \%, p=0.195)$ & $0.38(0.36,0.40)$ & 95.58 \\
\hline \multicolumn{3}{|l|}{ Heterogenety between groups: $p=0.658$} \\
\hline \multirow[t]{2}{*}{ Overall $(1$-squared $=53.1 \%, p=0.010)$} & $0.38(0.36,0.40)$ & 100.00 \\
\hline & & \\
\hline .01 & & \\
\hline
\end{tabular}

$\mathrm{F}$

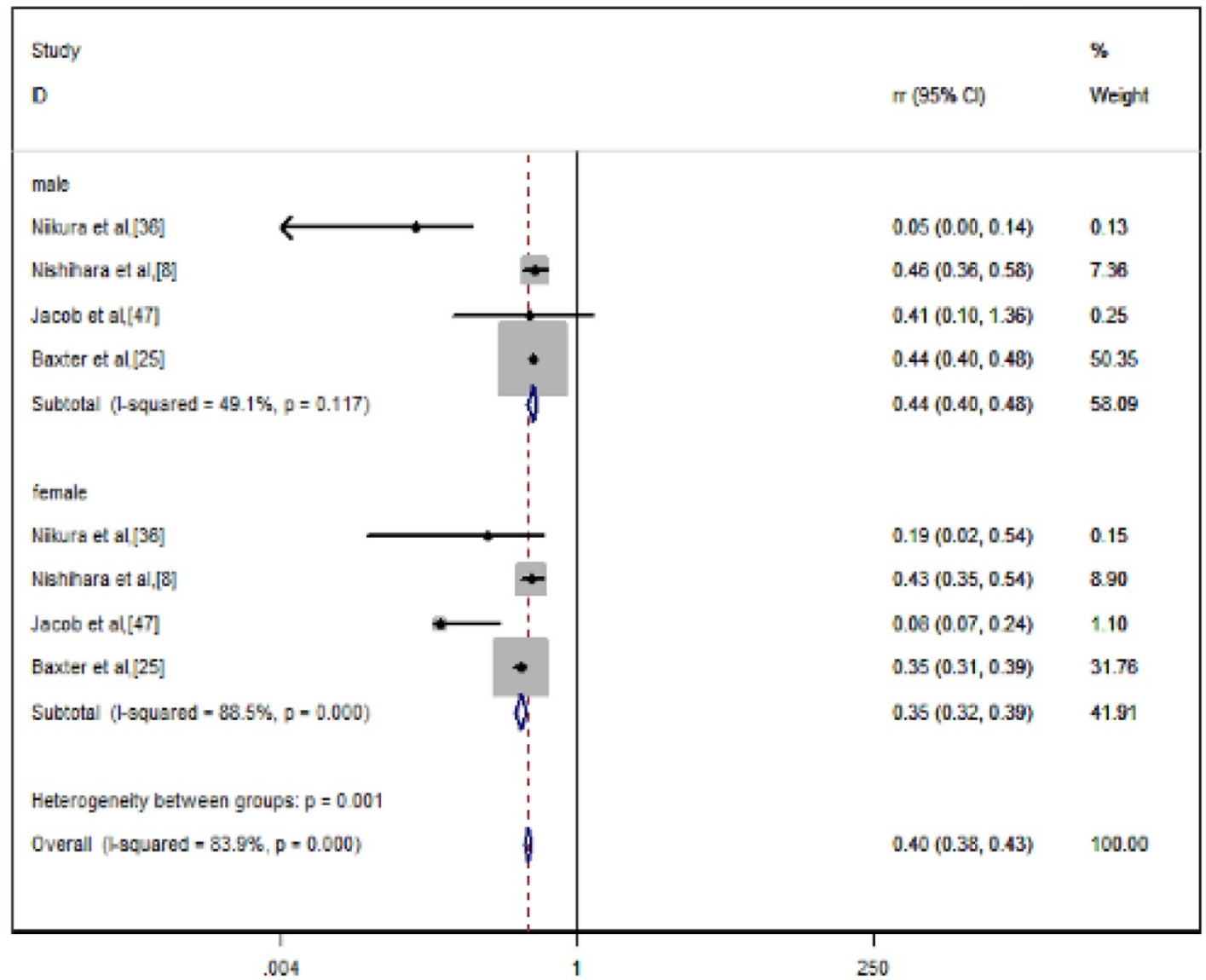

Figure 5. Colonoscopy was associated with a reduced morality of CRC in a subgroup analysis of forest plots; (A) Intervention; (B) Study design; (C) Country; (D) Sample size; (E) Age; (F) Sex. 

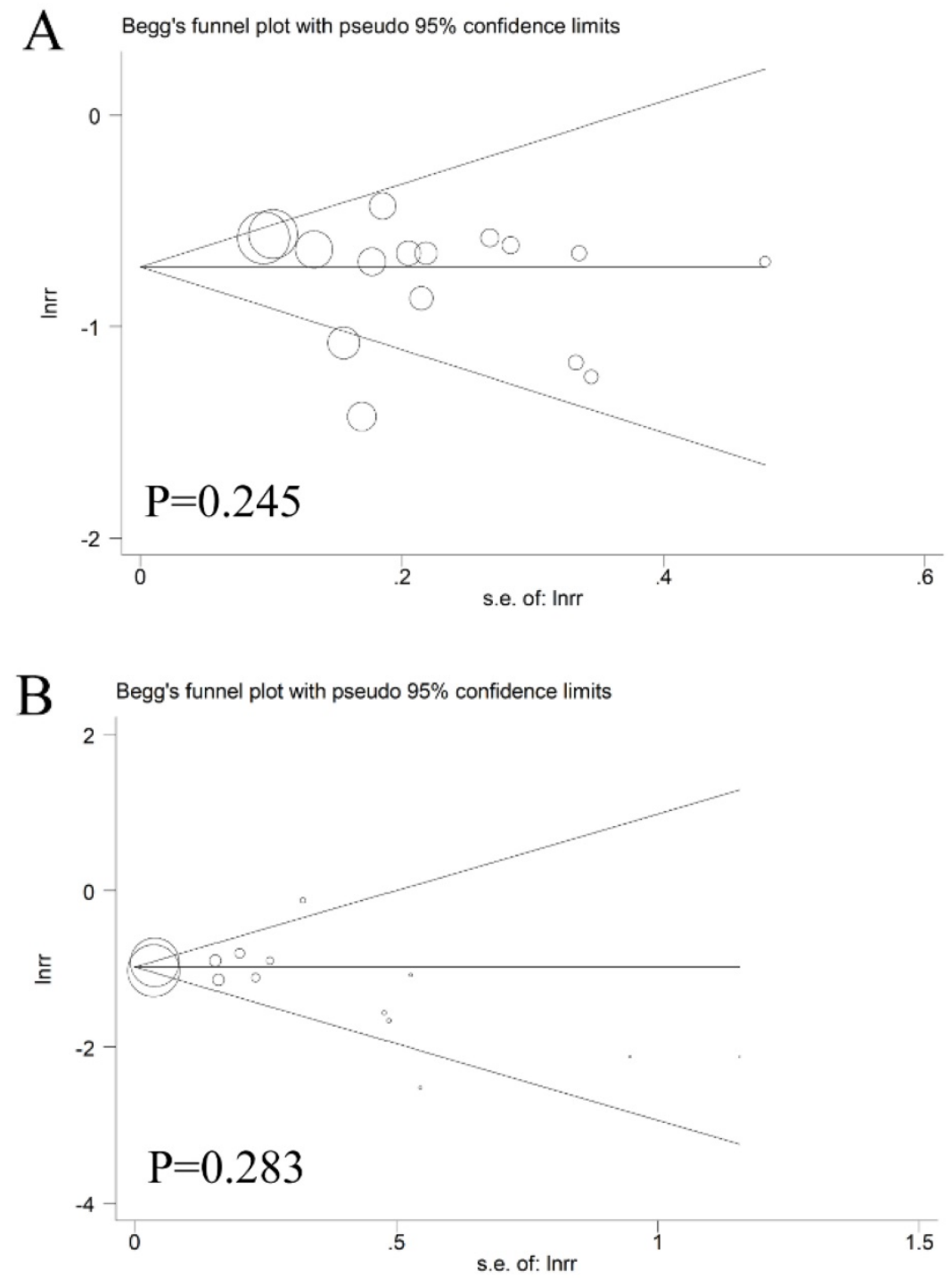

Figure 6. The Begg's funnel plot of the publication bias; (A) incidence; (B) mortality.

Table 4. Subgroup analysis of CRC mortality reduction after endoscopic screening

\begin{tabular}{|c|c|c|c|c|c|c|}
\hline \multirow{2}{*}{ Subgroups } & \multirow{2}{*}{ No. of studies } & \multirow{2}{*}{ Pooled RR (95\% CI) } & \multirow[t]{2}{*}{ Z } & \multirow[t]{2}{*}{$P$} & \multicolumn{2}{|c|}{ Heterogeneity } \\
\hline & & & & & I2 (\%) & $\mathrm{Ph}$ \\
\hline Intervention & & & & & & 3.34 \\
\hline Screening $[8,27,35,37,39,45,46,53,55,56]$ & 10 & $0.362(0.339-0.386)$ & 12.11 & 0.000 & 31.0 & \\
\hline Screening/diagnostic and Various* $[24,25,36,47]$ & 4 & $0.397(0.369-0.427)$ & 5.09 & 0.008 & 73.5 & \\
\hline Study design & & & & & & 5.99 \\
\hline Cochort $[8,35,36,37,39,45-47]$ & 8 & $0.356(0.333-0.381)$ & 9.89 & 0.000 & 45.0 & \\
\hline (Nested) case-control $[24,25,27,53,55,56]$ & 6 & $0.402(0.375-0.432)$ & 7.87 & 0.000 & 44.5 & \\
\hline Country & & & & & & 8.08 \\
\hline Western $[8,24,25,27,35,37,39,45-47,53,55,56]$ & 13 & $0.378(0.360-0.397)$ & 18.09 & 0.000 & 38.9 & \\
\hline Asia [36] & 1 & $0.080(0.027-0.233)$ & 4.63 & 0.000 & NA & \\
\hline Sample size & & & & & & 3.97 \\
\hline$\leq 10,000[27,39,53,55,56]$ & 5 & $0.409(0.311-0.537)$ & 4.06 & 0.000 & 54.4 & \\
\hline $10,000-100,000[8,24,25,36,45,46]$ & 6 & $0.394(0.368-0.423)$ & 8.94 & 0.000 & 58.1 & \\
\hline$\geq 1$ million $[35,38,47]$ & 3 & $0.358(0.334-0.384)$ & 4.62 & 0.000 & 34.5 & \\
\hline Age & & & & & & 0.20 \\
\hline $20-50[8,35,36,39,53,55]$ & 6 & $0.358(0.284-0.451)$ & 4.07 & 0.000 & 71.7 & \\
\hline$\geq 50[24,25,27,38,45-47,56]$ & 8 & $0.378(0.360-0.397)$ & 21.95 & 0.001 & 29.2 & \\
\hline Sex & & & & & & 11.47 \\
\hline Male $[8,25,36,47]$ & 4 & $0.440(0.404-0.479)$ & 7.00 & 0.000 & 49.1 & \\
\hline Female $[8,25,36,47]$ & 4 & $0.351(0.318-0.388)$ & 5.62 & 0.000 & 88.5 & \\
\hline
\end{tabular}

$\mathrm{NA}=$ not applicable; $\mathrm{RR}=$ relative risk $; \mathrm{CI}=$ confidence interval. 
As far as we know, this systematic review and meta-analysis might be one of the leading few studies assessing the value of colonoscopy screening in reducing the risk of CRC among healthy individuals. Moreover, we had observed good results among the associations between colonoscopy screening and the mortality and incidence of CRC. Of all the twentynine studies enrolled, only one was from the eastern country. For the remaining 28 studies, we found that colonoscopy could achieve $51 \% R R$ and $62 \% R R$ reduction in CRC incidence $(R R=0.49,95 \% C I=$ $0.45-0.53)$ and morality $(R R=0.38,95 \% C I=0.36-0.40)$ in western countries, which might be a reference for eastern countries. Further prospective studies from China, Japan and Korea are warranted.

Furthermore, the minimum age of regular colonoscopic screening is 50, recommended by developed countries $[19,58-60]$. It is unclear whether the population under 50 years old could be monitored in the same manner or not. Our study makes up for this gap. The population aged 20-50 years old who underwent colonoscopy was statistically analyzed. We found that colonoscopy could also achieve $64 \%$ $R R$ and $51 \% R R$ reduction in CRC mortality $(R R=$ $0.36,95 \% C I=0.28-0.45 ; p \leq 0.001)$ and incidence $(R R=$ $0.49,95 \% C I=0.43-0.56, p \leq 0.001)$. We found that colonoscopy screening had a similar protective effect on young people under the age of 50. And we need more data to draw more reliable conclusions.

What's more, direct access colonoscopy service for CRC screening produces a positive financial benefit for patients and local health districts [61]. As a clinician, based on our experience, colonoscopy can detect cancer early and have a positive effect on the prognosis of patients, although early colonoscopy is more expensive. However, its cost is lower in the long run, compared with the treatment of advanced cancer. At the same time, from the perspective of social development, it can reduce the direct cost and bring direct economic benefits. For example, colonoscopy early detection, early diagnosis of $\mathrm{CRC}$, patients can receive early treatment, so that he/she can work properly, will increase productivity, bring indirect economic benefits; if the patient does not work, it will increase leisure time. Regardless of the fact that this was not a quantifiable economic benefit, it may be an overall health benefit. These are all pertinent particularly to poorer, developing countries where resources are restricted.

In explaining our findings, attention should be also paid to the following aspects. On the one hand, we did not include randomized controlled trials (RCTs), because it was difficult to conduct RCTs, especially in Japan and Europe, where CRC screening has been introduced into national health programs.
What's more, colonoscopy utilization has been on the rise in North America [62-64] and some European countries [65], there are no RCTs results of CRC mortality. The best source of evidence for the reduction in CRC mortality after colonoscopy may be observational studies. On the other hand, the inevitable time deviation may have a certain impact on the assessment of mortality and incidence. Last but not least, some biases are inevitable in observational studies, especially self-selection bias. For example, in the exposure group and the control group of cohort or case-control studies, health-conscious people may receive colonoscopy compared to those who are not, which may overestimate the protective effect of colonoscopy.

Although sensitivity analyses partially explained heterogeneity, the primary source of the heterogeneity is unclear. It could be potentially generated by the inherent relationship between cancer occurrence and the pattern or frequency of colonoscopic inspection. Despite the use of a fixed-effects model in this analysis, it is noteworthy that estimates with high heterogeneity are vulnerable.

\section{Conclusions}

The results indicated that colonoscopy could significantly reduce the incidence and mortality of CRC. After subgroup analysis of different interventions, study design, country, sample size, age or sex, the outcomes remained consistent. Usually, the recommended age by developed countries for regular colonoscopic screening is 50 . Based on our results, the population aged 20-50 years old could also benefit from colonoscopic screening. Further researches were required to verify our findings.

\section{Supplementary Material}

Supplementary tables.

http://www.jcancer.org/v11p5953s1.pdf

\section{Acknowledgement}

This work was supported by China National Science and Technology major projects 13th 5-year plan (No.2018ZX10725505), and Innovation team project of Beijing University of Chinese Medicine (2019-JYB-TD-009).

\section{Author Contributions}

Conceptualization: JX Zhang, G Chen, YA Ye; Data curation: $P$ Zhang, $X$ Cao; Formal analysis: XK $\mathrm{Li}$, ZG Li, LD Zhang, DN Gan, Hongbo Du; Methodology: G Chen; Software: JX Zhang; Visualization: G Chen, JY Zhang; Writing-original draft: JX Zhang, G Chen. 


\section{Competing Interests}

The authors have declared that no competing interest exists.

\section{References}

1. Brody H. Colorectal cancer. Nature. 2015; 521: S1.

2. Petrelli F, Labianca R, Zaniboni A, Lonardi S, Galli F, Rulli E, et al. Assessment of Duration and Effects of 3 vs 6 Months of Adjuvant Chemotherapy in High-Risk Stage II Colorectal Cancer: A Subgroup Analysis of the TOSCA Randomized Clinical Trial. JAMA oncology. 2020;6(4):547-551.

3. Weitz J, Koch M, Debus J, Hohler T, Galle PR, Buchler MW. Colorectal cancer. Lancet (London, England). 2005; 365: 153-65.

4. Jemal A, Siegel R, Ward E, Hao Y, Xu J, Thun MJ. Cancer statistics, 2009. CA: a cancer journal for clinicians. 2009; 59: 225-49.

5. Expert Consensus on early Colorectal cancer screening Process in China (2019, Shanghai). Chinese Journal of Digestive endoscopy. 2019;10: 709-19. (in Chinese)

6. Jemal A, Bray F, Center MM, Ferlay J, Ward E, Forman D. Global cancer statistics. CA: a cancer journal for clinicians. 2011; 61: 69-90.

7. Rapuri S, Spencer J, Eckels D. Importance of postpolypectomy surveillance and postpolypectomy compliance to follow-up screening--review of literature. International journal of colorectal disease. 2008; 23: 453-9.

8. Nishihara R, Wu K, Lochhead P, Morikawa T, Liao X, Qian ZR, et al. Long-term colorectal-cancer incidence and mortality after lower endoscopy. The New England journal of medicine. 2013; 369: 1095-105.

9. Brenner $\mathrm{H}$, Haug $\mathrm{U}$, Arndt $\mathrm{V}$, Stegmaier $\mathrm{C}$, Altenhofen $\mathrm{L}$, Hoffmeister M. Low risk of colorectal cancer and advanced adenomas more than 10 years after negative colonoscopy. Gastroenterology. 2010; 138: 870-6.

10. Schoen RE, Pinsky PF, Weissfeld JL, Yokochi LA, Church T, Laiyemo AO, et al. Colorectal-cancer incidence and mortality with screening flexible sigmoidoscopy. The New England journal of medicine. 2012; 366: 2345-57.

11. Kahi CJ, Imperiale TF, Juliar BE, Rex DK. Effect of screening colonoscopy on colorectal cancer incidence and mortality. Clinical gastroenterology and hepatology : the official clinical practice journal of the American Gastroenterological Association. 2009; 7: 770-5; quiz 11.

12. Kavanagh AM, Giovannucci EL, Fuchs CS, Colditz GA. Screening endoscopy and risk of colorectal cancer in United States men. Cancer causes \& control : CCC. 1998; 9: 455-62.

13. Brenner H, Chang-Claude J, Seiler CM, Hoffmeister M. Long-term risk of colorectal cancer after negative colonoscopy. Journal of clinical oncology : official journal of the American Society of Clinical Oncology. 2011; 29: 3761-7.

14. Brenner H, Chang-Claude J, Seiler CM, Rickert A, Hoffmeister M. Protection from colorectal cancer after colonoscopy: a population-based, case-control study. Annals of internal medicine. 2011; 154: 22-30.

15. Sung JJ, Ng SC, Chan FK, Chiu HM, Kim HS, Matsuda T, et al. An updated Asia Pacific Consensus Recommendations on colorectal cancer screening. Gut. 2015; 64: 121-32

16. Kaminski MF, Bretthauer M, Zauber AG, Kuipers EJ, Adami HO, van Ballegooijen $\mathrm{M}$, et al. The NordICC Study: rationale and design of a randomized trial on colonoscopy screening for colorectal cancer. Endoscopy. 2012; 44: 695-702.

17. Colonoscopy versus Fecal Immunochemical Testing in Colorectal-Cancer Screening. The New England journal of medicine. 2016; 374: 1898.

18. Sali L, Grazzini G, Carozzi F, Castiglione G, Falchini M, Mallardi B, et al. Screening for colorectal cancer with FOBT, virtual colonoscopy and optical colonoscopy: study protocol for a randomized controlled trial in the Florence district (SAVE study). Trials. 2013; 14: 74.

19. von Karsa L, Patnick J, Segnan N. European guidelines for quality assurance in colorectal cancer screening and diagnosis. First Edition--Executive summary. Endoscopy. 2012; 44 Suppl 3: Se1-8.

20. Altobelli E, Lattanzi A, Paduano R, Varassi G, di Orio F. Colorectal cancer prevention in Europe: burden of disease and status of screening programs. Preventive medicine. 2014; 62: 132-41.

21. Meester RG, Doubeni CA, Zauber AG, Goede SL, Levin TR, Corley DA, et al. Public health impact of achieving $80 \%$ colorectal cancer screening rates in the United States by 2018. Cancer. 2015; 121: 2281-5.

22. Brenner H, Altenhofen L, Stock C, Hoffmeister M. Expected long-term impact of the German screening colonoscopy programme on colorectal cancer prevention: analyses based on 4,407,971 screening colonoscopies. European journal of cancer (Oxford, England : 1990). 2015; 51: 1346-53.

23. Baxter NN, Goldwasser MA, Paszat LF, Saskin R, Urbach DR, Rabeneck L. Association of colonoscopy and death from colorectal cancer. Annals of internal medicine. 2009; 150: 1-8.

24. Muller AD, Sonnenberg A. Protection by endoscopy against death from colorectal cancer. A case-control study among veterans. Archives of internal medicine. 1995; 155: 1741-8.

25. Baxter NN, Warren JL, Barrett MJ, Stukel TA, Doria-Rose VP. Association between colonoscopy and colorectal cancer mortality in a US cohort according to site of cancer and colonoscopist specialty. Journal of clinical oncology : official journal of the American Society of Clinical Oncology. 2012; 30: 2664-9.
26. Singh $\mathrm{H}$, Nugent $Z$, Demers AA, Kliewer EV, Mahmud SM, Bernstein CN. The reduction in colorectal cancer mortality after colonoscopy varies by site of the cancer. Gastroenterology. 2010; 139: 1128-37.

27. Doubeni CA, Corley DA, Quinn VP, Jensen CD, Zauber AG, Goodman M, et al. Effectiveness of screening colonoscopy in reducing the risk of death from right and left colon cancer: a large community-based study. Gut. 2018; 67: 291-8

28. Stroup DF, Berlin JA, Morton SC, Olkin I, Williamson GD, Rennie D, et al. Meta-analysis of observational studies in epidemiology: a proposal for reporting. Meta-analysis Of Observational Studies in Epidemiology (MOOSE) group. Jama. 2000; 283: 2008-12

29. Moher D, Liberati A, Tetzlaff J, Altman DG. Reprint--preferred reporting items for systematic reviews and meta-analyses: the PRISMA statement. Physical therapy. 2009; 89: 873-80.

30. DerSimonian R, Laird N. Meta-analysis in clinical trials. Control Clin Trials. 1986; $7: 177-88$.

31. Higgins JP, Thompson SG, Deeks JJ, Altman DG. Measuring inconsistency in meta-analyses. BMJ (Clinical research ed). 2003; 327: 557-60

32. Begg CB, Mazumdar M. Operating characteristics of a rank correlation test for publication bias. Biometrics. 1994; 50: 1088-101.

33. Egger M, Davey Smith G, Schneider M, Minder C. Bias in meta-analysis detected by a simple, graphical test. Bmj. 1997; 315: 629-34.

34. Duval S, Tweedie R. Trim and fill: A simple funnel-plot-based method of testing and adjusting for publication bias in meta-analysis. Biometrics. 2000; 56: 455-63.

35. Lee JK, Jensen CD, Levin TR, Zauber AG, Schottinger JE, Quinn VP, et al. Long-term Risk of Colorectal Cancer and Related Deaths After a Colonoscopy With Normal Findings. JAMA internal medicine. 2019; 179: 153-60.

36. Niikura R, Hirata Y, Suzuki N, Yamada A, Hayakawa Y, Suzuki H, et al. Colonoscopy reduces colorectal cancer mortality: A multicenter, long-term, colonoscopy-based cohort study. PloS one. 2017; 12: e0185294.

37. Wang YR, Cangemi JR, Loftus EV, Jr., Picco MF. Decreased Risk of Colorectal Cancer after Colonoscopy in Patients 76-85 Years Old in the United States. Digestion. 2016; 93: 132-8.

38. Stock D, Paszat LF, Rabeneck L. Colorectal cancer mortality reduction is associated with having at least 1 colonoscopy within the previous 10 years among a population-wide cohort of screening age. Gastrointestinal endoscopy. 2016; 84: 133-41.

39. Ananthakrishnan AN, Cagan A, Cai T, Gainer VS, Shaw SY, Churchill S, et al. Colonoscopy is associated with a reduced risk for colon cancer and mortality in patients with inflammatory bowel diseases. Clinical gastroenterology and hepatology : the official clinical practice journal of the American Gastroenterological Association. 2015; 13: 322-9.e1.

40. Kahi CJ, Myers LJ, Slaven JE, Haggstrom D, Pohl H, Robertson DJ, et al. Lower endoscopy reduces colorectal cancer incidence in older individuals. Gastroenterology. 2014; 146: 718-25.e3.

41. Morois S, Cottet V, Racine A, Clavel-Chapelon F, Carbonnel F, Bastide N, et al. Colonoscopy reduced distal colorectal cancer risk and excess cancer risk associated with family history. Cancer causes \& control : CCC. 2014; 25: 1329-36.

42. Brenner H, Chang-Claude J, Jansen L, Knebel P, Stock C, Hoffmeister M. Reduced risk of colorectal cancer up to 10 years after screening, surveillance, or diagnostic colonoscopy. Gastroenterology. 2014; 146: 709-17.

43. Doubeni CA, Weinmann S, Adams K, Kamineni A, Buist DS, Ash AS, et al. Screening colonoscopy and risk for incident late-stage colorectal cancer diagnosis in average-risk adults: a nested case-control study. Annals of internal medicine. 2013; 158: 312-20.

44. Wang YR, Cangemi JR, Loftus EV, Jr., Picco MF. Risk of colorectal cancer after colonoscopy compared with flexible sigmoidoscopy or no lower endoscopy among older patients in the United States, 1998-2005. Mayo Clinic proceedings. 2013; 88: 464-70.

45. Eldridge RC, Doubeni CA, Fletcher RH, Zauber AG, Corley DA, Doria-Rose $\mathrm{VP}$, et al. Uncontrolled confounding in studies of screening effectiveness: an example of colonoscopy. Journal of medical screening. 2013; 20: 198-207.

46. Manser CN, Bachmann LM, Brunner J, Hunold F, Bauerfeind P, Marbet UA Colonoscopy screening markedly reduces the occurrence of colon carcinomas and carcinoma-related death: a closed cohort study. Gastrointestinal endoscopy. 2012; 76: 110-7.

47. Jacob BJ, Moineddin R, Sutradhar R, Baxter NN, Urbach DR. Effect of colonoscopy on colorectal cancer incidence and mortality: an instrumental variable analysis. Gastrointestinal endoscopy. 2012; 76: 355-64.e1.

48. Mulder SA, van Soest EM, Dieleman JP, van Rossum LG, Ouwendijk RJ, van Leerdam ME, et al. Exposure to colorectal examinations before a colorectal cancer diagnosis: a case-control study. European journal of gastroenterology \& hepatology. 2010; 22: 437-43.

49. Blom J, Yin L, Liden A, Dolk A, Jeppsson B, Pahlman L, et al. A 9-year follow-up study of participants and nonparticipants in sigmoidoscopy screening: importance of self-selection. Cancer epidemiology, biomarkers \& prevention : a publication of the American Association for Cancer Research, cosponsored by the American Society of Preventive Oncology. 2008; 17: 1163-8.

50. Cotterchio M, Manno M, Klar N, McLaughlin J, Gallinger S. Colorectal screening is associated with reduced colorectal cancer risk: a case-control study within the population-based Ontario Familial Colorectal Cancer Registry. Cancer causes \& control : CCC. 2005; 16: 865-75 
51. Newcomb PA, Storer BE, Morimoto LM, Templeton A, Potter JD. Long-term efficacy of sigmoidoscopy in the reduction of colorectal cancer incidence. Journal of the National Cancer Institute. 2003; 95: 622-5.

52. Slattery ML, Edwards SL, Ma KN, Friedman GD. Colon cancer screening, lifestyle, and risk of colon cancer. Cancer causes \& control : CCC. 2000; 11: 555-63.

53. Scheitel SM, Ahlquist DA, Wollan PC, Hagen PT, Silverstein MD. Colorectal cancer screening: a community case-control study of proctosigmoidoscopy, barium enema radiography, and fecal occult blood test efficacy. Mayo Clinic proceedings. 1999; 74: 1207-13.

54. Muller AD, Sonnenberg A. Prevention of colorectal cancer by flexible endoscopy and polypectomy. A case-control study of 32,702 veterans. Annals of internal medicine. 1995; 123: 904-10.

55. Selby JV, Friedman GD, Quesenberry CP, Jr., Weiss NS. A case-control study of screening sigmoidoscopy and mortality from colorectal cancer. The New England journal of medicine. 1992; 326: 653-7.

56. Newcomb PA, Norfleet RG, Storer BE, Surawicz TS, Marcus PM. Screening sigmoidoscopy and colorectal cancer mortality. Journal of the National Cancer Institute. 1992; 84: 1572-5

57. Ko CW, Doria-Rose VP, Barrett MJ, Kamineni A, Enewold L, Weiss NS. Screening colonoscopy and flexible sigmoidoscopy for reduction of colorectal cancer incidence: A case-control study. PloS one. 2019; 14: e0226027.

58. Leddin D, Hunt R, Champion M, Cockeram A, Flook N, Gould M, et al. Canadian Association of Gastroenterology and the Canadian Digestive Health Foundation: Guidelines on colon cancer screening. Canadian journal of gastroenterology = Journal canadien de gastroenterologie. 2004; 18: 93-9.

59. Rex DK, Johnson DA, Anderson JC, Schoenfeld PS, Burke CA, Inadomi JM. American College of Gastroenterology guidelines for colorectal cancer screening 2009 [corrected]. The American journal of gastroenterology. 2009; 104: 739-50.

60. Levin B, Lieberman DA, McFarland B, Andrews KS, Brooks D, Bond J, et al. Screening and surveillance for the early detection of colorectal cancer and adenomatous polyps, 2008: a joint guideline from the American Cancer Society, the US Multi-Society Task Force on Colorectal Cancer, and the American College of Radiology. Gastroenterology. 2008; 134: 1570-95.

61. Clarke L, Pockney P, Gillies D, Foster R, Gani J. Direct access colonoscopy service for bowel cancer screening produces a positive financial benefit for patients and local health districts. Internal medicine journal. 2019; 49: 729-33.

62. Stock C, Haug U, Brenner H. Population-based prevalence estimates of history of colonoscopy or sigmoidoscopy: review and analysis of recent trends. Gastrointestinal endoscopy. 2010; 71: 366-81.e2

63. Rabeneck L, Paszat LF, Saskin R, Stukel TA. Association between colonoscopy rates and colorectal cancer mortality. The American journal of gastroenterology. 2010; 105: 1627-32.

64. Lieberman DA, Williams JL, Holub JL, Morris CD, Logan JR, Eisen GM, et al. Colonoscopy utilization and outcomes 2000 to 2011. Gastrointestinal endoscopy. 2014; 80: 133-43.

65. Zavoral M, Suchanek S, Zavada F, Dusek L, Muzik J, Seifert B, et al Colorectal cancer screening in Europe. World journal of gastroenterology. 2009; 15: 5907-15 